\title{
Radiation forces constrain the FRB mechanism
}

\author{
Pawan Kumar $^{1 \star}$ and Wenbin Lu ${ }^{\circledR 2 \star}$ \\ ${ }^{1}$ Department of Astronomy, University of Texas at Austin, Austin, TX 78712, USA \\ ${ }^{2}$ TAPIR, Walter Burke Institute for Theoretical Physics, Caltech, Mail Code 350-17, Pasadena, CA 91125, USA
}

Accepted 2020 March 19. Received 2020 March 9; in original form 2019 December 9

\begin{abstract}
We provide constraints on fast radio burst (FRB) models by careful considerations of radiation forces associated with these powerful transients. We find that the induced Compton scatterings of the coherent radiation by electrons/positrons accelerate particles to very large Lorentz factors (LFs) in and around the source of this radiation. This severely restricts those models for FRBs that invoke relativistic shocks and maser-type instabilities at distances less than about $10^{13}$ $\mathrm{cm}$ of the neutron star. Radiation travelling upstream, in these models, forces particles to move away from the shock with an LF larger than the LF of the shock front. This suspends the photon generation process after it has been operating for less than $\sim 0.1 \mathrm{~ms}$ (observer frame duration). We show that masers operating in shocks at distances larger than $10^{13} \mathrm{~cm}$ cannot simultaneously account for the burst duration of $1 \mathrm{~ms}$ or more and the observed $\sim \mathrm{GHz}$ frequencies of FRBs without requiring an excessive energy budget $\left(>10^{46} \mathrm{erg}\right.$ ); the energy is not calculated by imposing any efficiency consideration, or other details, for the maser mechanism, but is entirely the result of ensuring that particle acceleration by induced Compton forces upstream of the shock front does not choke off the maser process. For the source to operate more or less continuously for a few ms, it should be embedded in a strong magnetic field - cyclotron frequency $\gg$ wave frequency - so that radiation forces do not disperse the plasma and shut off the engine.
\end{abstract}

Key words: masers - radiation mechanisms: non-thermal-methods: analytical-stars: magnetars.

\section{INTRODUCTION}

Fast radio bursts (FRBs) are millisecond duration bright (flux density $\sim$ Jy) transient events observed between $400 \mathrm{MHz}$ and $7 \mathrm{GHz}$ frequencies (Lorimer et al. 2007; Thornton et al. 2013; Spitler et al. 2014, 2016; Petroff et al. 2016; Bannister et al. 2017, 2019; Chatterjee et al. 2017; Law et al. 2017; Marcote et al. 2017; Tendulkar et al. 2017; Farah et al. 2018; Gajjar et al. 2018; Michilli et al. 2018; Shannon et al. 2018; Amiri et al. 2019a, b; Kocz et al. 2019; Oslowski et al. 2019; Ravi 2019a, b; Ravi et al. 2019). Numerous mechanisms have been suggested for the generation of the coherent radio emission of FRBs (e.g. Kumar, Lu \& Bhattacharya 2017; Metzger, Berger \& Margalit 2017; Lu \& Kumar 2018; Yang \& Zhang 2018; Metzger, Margalit \& Sironi 2019; for recent reviews, see Katz 2018; Cordes \& Chatterjee 2019; Petroff, Hessels \& Lorimer 2019). Most of these mechanisms are proposed to operate at a distance from neutron star surface of $10^{13}$ $\mathrm{cm}$ or less. We explore in this paper constraints on the FRB radiation mechanisms provided by rapid acceleration of charge particles by the strong electric field associated with the radiation and induced Compton (IC) scatterings of photons; IC scattering refers to the scattering of photons by electrons when the occupation number of photon quantum states is much larger than unity (for FRBs, the occupation number is of the order of $10^{35}$ ). The electronphoton scattering optical depth is increased due to the IC effect, which has been calculated by many authors, e.g. Melrose (1971), Blandford (1973), Blandford \& Scharlemann (1975), Blandford \& Scharlemann (1976), and Wilson \& Rees (1978). We include the constraint on FRB models provided by IC optical depth, but that turns out to be much weaker than the IC acceleration that is estimated here. Particle acceleration due to the electric field of FRB radiations and its implications are considered in Section 2. Acceleration due to IC scatterings is described in Section 3, and the constraints these processes and some other general considerations impose on the FRB source and radiation mechanisms are discussed in Section 4.

\section{PARTICLE MOTION DUE TO LARGE AMPLITUDE EM WAVE AND STATIC MAGNETIC FIELD}

The rms electric field strength associated with FRB radiation at a distance $R$ from the source is

$$
\frac{E_{0}}{\sqrt{2}}=\left(\frac{L}{c R^{2}}\right)^{1 / 2}=\left(1.8 \times 10^{3} \mathrm{esu}\right) L_{43}^{1 / 2} R_{13}^{-1},
$$


where $L$ is isotropic equivalent luminosity of the FRB. The nonlinearity parameter, $a$, associated with this field - which is a rough measure of the energy gained by an electron travelling a distance of one wavelength in the wave electric field divided by its rest mass energy - is

$a \equiv \frac{q E_{0}}{m c \omega}=4.5 L_{43}^{1 / 2} R_{13}^{-1} \omega_{10}^{-1}$,

where $q$ and $m$ are electron charge and mass, respectively, and $\omega$ is the wave frequency $\left(\mathrm{rad} \mathrm{s}^{-1}\right)$.

Let us consider the motion of a particle exposed to a linearly polarized electromagnetic (EM) wave and a uniform magnetic field that is perpendicular to the wave vector of the EM wave. The wave EM fields and vector potentials for the EM wave and the static magnetic field are as follows:

$\mathbf{E}_{\mathrm{w}}=E_{0} \hat{\mathbf{x}} \sin (k z-\omega t), \quad \mathbf{B}_{\mathrm{w}}=E_{0} \hat{\mathbf{y}} \sin (k z-\omega t)$,

$\mathbf{A}_{\mathrm{w}}=-\frac{c E_{0}}{\omega} \hat{\mathbf{x}} \cos (k z-\omega t), \quad \mathbf{A}_{\mathrm{B}}=\left(B_{y} \hat{\mathbf{x}}-B_{x} \hat{\mathbf{y}}\right) z$.

The Lagrangian for particle motion is

$L=-\frac{m c^{2}}{\gamma}+\frac{q}{c} \mathbf{A} \cdot \mathbf{v}$,

where $\gamma$ is the Lorentz factor (LF) of the particle, $\mathbf{v}$ is its 3velocity, and $\mathbf{A}=\mathbf{A}_{\mathrm{w}}+\mathbf{A}_{\mathrm{B}}$ is the vector potential for the EM wave plus the static magnetic field. The $x$ and $y$ components of the particle's canonical momentum are conserved since the Lagrangian is independent of $x$ and $y$ coordinates:

$p_{x}=m \gamma v_{x}+\frac{q A_{x}}{c}=m \gamma v_{x}-\frac{q E_{0}}{\omega} \cos \psi+\frac{q B_{y} z}{c}=$ constant,

$p_{y}=m \gamma v_{y}-\frac{q B_{x} z}{c}=$ constant,

where

$\psi=k z-\omega t$.

The $z$-component of the momentum equation

$\frac{\mathrm{d} \gamma v_{z}}{\mathrm{~d} t}=\frac{q}{m c}\left[B_{\mathrm{w}} v_{x}+v_{x} B_{y}-v_{y} B_{x}\right]$

can be rewritten as

$\frac{\mathrm{d}}{\mathrm{d} t}\left[\gamma v_{z}-\gamma c-\omega_{\mathrm{B}}\left(x \sin \theta_{\mathrm{B}}-y \cos \theta_{\mathrm{B}}\right)\right]=0$,

where

$\sin \theta_{\mathrm{B}}=B_{y} / B \quad$ and $\quad \omega_{\mathrm{B}}=\frac{q B}{m c}$

is the cyclotron frequency.

We assume that the particle is initially at rest, i.e. $\mathbf{v}=\mathbf{0}$, before it is hit by the EM wave, and that its initial position is $\mathbf{r}=\mathbf{0}$. Thereafter, its velocity is obtained from equations (6), (7), and (10) by applying the initial conditions

$\gamma v_{x}=a c[\cos (k z-\omega t)-1]-\omega_{\mathrm{B}} z \sin \theta_{\mathrm{B}}$,

$\gamma v_{y}=\omega_{\mathrm{B}} z \cos \theta_{\mathrm{B}}$

$\gamma v_{z}=c(\gamma-1)+\omega_{\mathrm{B}}\left(x \sin \theta_{\mathrm{B}}-y \cos \theta_{\mathrm{B}}\right)$

where $a$ is given by equation (2).
The particle LF is easily obtained from these equations and is

$$
\begin{aligned}
\gamma= & \frac{4 a^{2} \sin ^{4}(\psi / 2)+4 a \sin ^{2}(\psi / 2) \xi_{z} \sin \theta_{\mathrm{B}}+\xi_{z}^{2}+1}{2\left[1-\xi_{\rho} \sin \left(\theta_{\mathrm{B}}-\phi\right)\right]} \\
& +\left[1-\xi_{\rho} \sin \left(\theta_{\mathrm{B}}-\phi\right)\right] / 2,
\end{aligned}
$$

where

$\sin \phi=y / \rho, \quad \rho^{2}=x^{2}+y^{2}, \quad \xi_{\rho}=\rho \omega_{\mathrm{B}} / c, \quad \xi_{z}=z \omega_{\mathrm{B}} / c$.

The particular case of $\omega_{\mathrm{B}}=0$, i.e. vanishing static magnetic field, is illuminating:

$\gamma v_{x}=-2 a c \sin ^{2}(\psi / 2), \gamma v_{z}=c(\gamma-1), \gamma=1+2 a^{2} \sin ^{4}(\psi / 2)$.

For $a>1$, the particle LF is of order $2 a^{2}$ and its velocity vector lies within an angle $\sim a^{-1}$ of the wave propagation direction. For $a \ll 1$, the velocity component along the wave vector oscillates at frequency $2 \omega$ and perpendicular to it at frequency $\omega$. However, for $a$ $>1$, the phase function along the particle worldline $|\psi|=\omega t-k z(t)$ $\sim \omega t / 2\left\langle\gamma^{2}\right\rangle$, and so the particle LF oscillates at frequency $\sim \omega /\langle\gamma$ $\left.{ }^{2}\right\rangle \sim \omega /\left[2\left(1+a^{2}\right)\right]$, where $\left\langle\gamma^{2}\right\rangle$ is the time-averaged LF-squared of the particle. Fig. 1 shows numerical results for particle trajectories for a few different values of $a$.

Particles out to a very large distance from the source of the intense FRB radiation are vigorously agitated and forced to move at high velocities - the time-averaged LF $\gamma \sim a^{2} \sim 10^{2} R_{13}^{-2}$; the non-linearity parameter for those FRBs that emit a good fraction of their energy at $\sim 500 \mathrm{MHz}$ frequency is $a \sim 10 R_{13}^{-1} L_{43}^{1 / 2}$ (see equation 2).

Considering that charge particles are forced to move at close to the speed of light by the electric field of the radiation out to a distance $\sim 10^{14} \mathrm{~cm}$ from the source, it might be hard for the plasma lens model (Cordes et al. 2017; Main et al. 2018) to operate within this distance of the source - a few ms duration radiation pulse cannot pass through the plasma in the 'lens' if the plasma is located at a distance $\lesssim 10^{13} \mathrm{~cm}$ from the FRB source since the plasma would be forced to move at speed $\sim c$ by the wave electric field - unless the magnification factor of the plasma lens is much larger than $\sim 10^{2}$.

If the FRB radiation is produced in shock-heated plasma (e.g. Metzger et al. 2019) at radius $R$, then particles upstream of the shock front would be accelerated to LF $\gamma \approx 2 a^{2} \sim 50 L_{43} R_{13}^{-2} \omega_{10}^{-2}$ (equation 2) by the electric field of radiation moving upstream as long as the composition of the medium is $\mathrm{e}^{ \pm}$and the cyclotron frequency is much less than $1 \mathrm{GHz}$. The LF of upstream particles with respect to the shock in this case is $\sim \gamma_{\mathrm{sf}} / 2 \gamma$, where $\gamma_{\mathrm{sf}}$ is the LF of the shock front with respect to the undisturbed, upstream, medium. The upstream medium is also compressed by a factor $\sim 4 a^{2}$ due to this acceleration, and the component of upstream particle 4-velocity tangential to the shock surface is $\sim a$, which varies on a time-scale of the wave period. These effects would modify the growth rate of synchrotron maser instability operating near the shock front, and reduce the efficiency of converting blast wave energy to coherent $\mathrm{GHz}$ radiation.

Furthermore, particle acceleration upstream of the shock front would shut off radiation production for a while in the observed band, if $R \lesssim 10^{13} \mathrm{~cm}$, until upstream particles slow down by plowing into the plasma further out. So, the radiation by shocked plasma is not going to be produced continuously if $R \lesssim 10^{13} \mathrm{~cm}$.

Another effect of particle acceleration by the intense FRB radiation is that it depletes the energy from the outward moving 

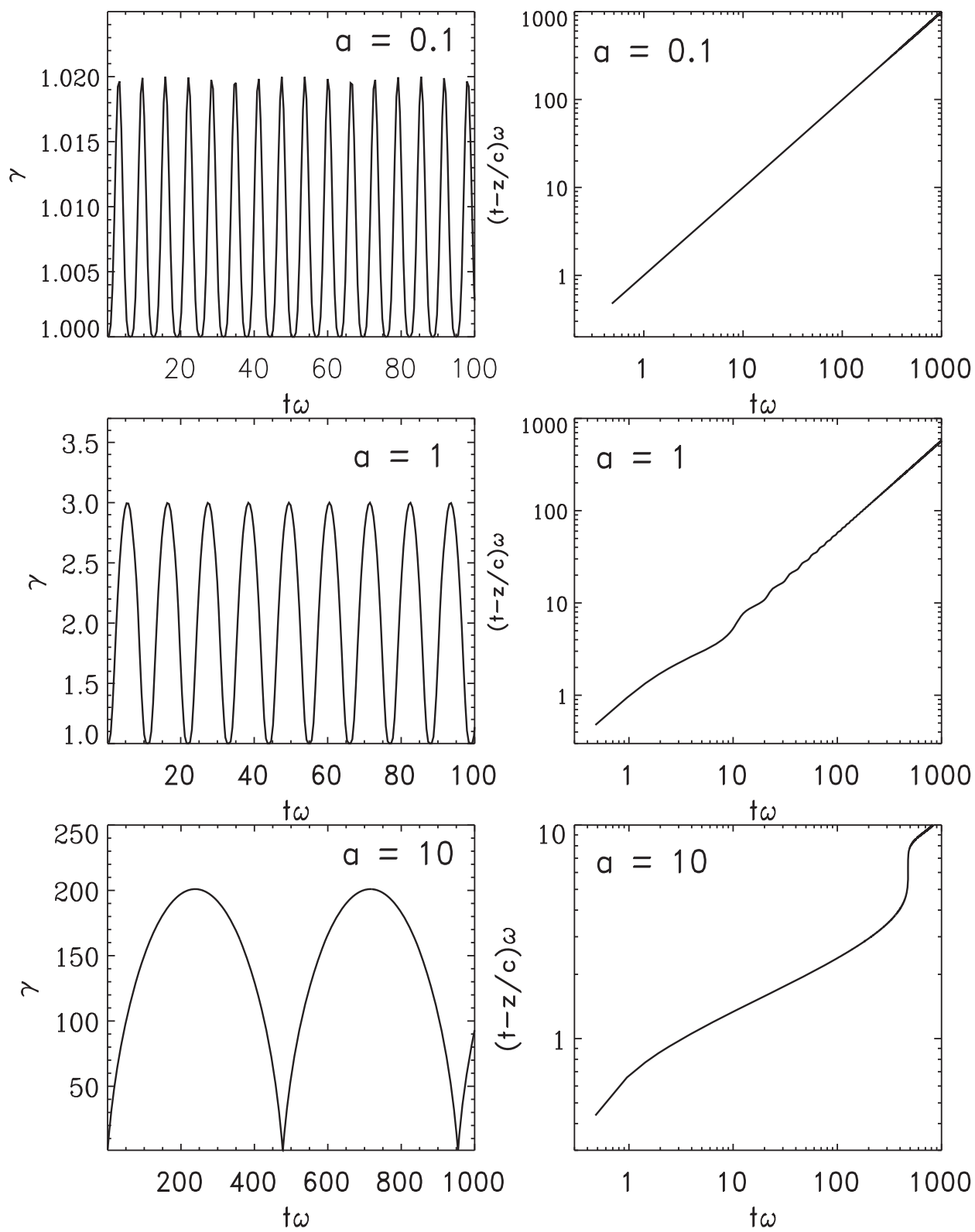

Figure 1. The left-hand panels show the particle LF, $\gamma$, as a function of dimensionless time, $t \omega$, for three different values of $a$ : $0.1,1$, and 10 (top to bottom), where $\omega$ is the EM wave frequency. These calculations are for weak magnetic fields such that the ratio of the electron cyclotron frequency and the EM wave frequency $\left(\omega_{\mathrm{B}} / \omega\right)$ is $10^{-5}$; results for $\omega_{\mathrm{B}} / \omega=10^{-3}$ are effectively the same as shown here. The three right-hand panels show the location of the particle with respect to the front of the EM wave, i.e. $[t-z(t) / c] \omega$; for large values of $a$, the particle rides the wave at a roughly constant phase angle for a long duration of time (see the bottom right panel for $a=10$ ).

radiation front - charge particles undergoing acceleration radiate - at a rate much larger than one might expect from Thomson scatterings. This is estimated in the following section, where we also discuss the constraints imposed by this process on the circumburst medium.

\subsection{Radiative loss of wave-accelerated particles}

We calculate in this section the energy loss suffered by FRB pulse as it travels through the circumstellar medium (CSM) of the magnetar due to power radiated by particles that are accelerated by the EM field of the FRB radiation (particle acceleration was calculated in Section 2). The power emitted by a relativistic particle undergoing acceleration is given by (the covariant form of) the Larmor formula

$$
P=\frac{2 q^{2}}{3 c}\left[-\left(\frac{\mathrm{d} u^{0}}{\mathrm{~d} \tau}\right)^{2}+\sum_{i=x, y, z}\left(\frac{\mathrm{d} u^{i}}{\mathrm{~d} \tau}\right)^{2}\right],
$$

where $\left(u^{\mu}\right)=\gamma\left(1, v_{x}, v_{y}, v_{z}\right)$ is the 4-velocity and $\mathrm{d} \tau=\mathrm{d} t / \gamma$ is the differential proper time. The total emitted energy within the interaction time $t_{\text {int }}$ is then given by $E_{\text {rad }}=\int_{0}^{t_{\text {int }}} P \mathrm{~d} t$, which is the amount of energy that is spontaneously scattered by the particle according to classical electrodynamics. In this picture, the scattered photons occupy very different regions of the phase space (for both frequency and direction) from the incoming photons, so we ignore stimulated emission (which will be discussed in the next 
section). We have checked that the radiative back-reaction force is negligible compared to the Lorentz force for the entire parameter space considered in this paper. However, as we show later, the cumulative energy loss could be significant such that a large fraction of the FRB wave energy is scattered away. This in turn provides a constraint on the gas density of the FRB environment.

Consider the FRB waves propagating through a strongly magnetized relativistic wind with luminosity $L_{\mathrm{w}}$ and $\mathrm{LF} \gamma_{\mathrm{w}}$. An order unity fraction of the wind power is carried by Poynting flux and a fraction $\mu_{\mathrm{w}}^{-1}<1$ is carried by electron (and positron) kinetic energy flux. At radius $R$ (much greater than the light cylinder of a neutron star), the magnetic field strength in the lab frame is $B \simeq \sqrt{L_{\mathrm{w}} /\left(r^{2} c\right)}$. For a pulse of duration $t_{\mathrm{FRB}}$, the interaction time between a particle and the FRB wave is

$t_{\text {int }} \simeq \min \left(R / c, \gamma_{\mathrm{w}}^{2} t_{\mathrm{FRB}}\right)$.

We calculate the cumulative scattered energy for each electron in the wind co-moving frame $E_{\mathrm{rad}}^{\prime}$ and then Lorentz transform that to the lab frame $E_{\mathrm{rad}}=\gamma_{\mathrm{w}} E_{\mathrm{rad}}^{\prime}$. The total number of electrons participating in the interaction near characteristic radius $R$ is

$N_{\mathrm{e}} \simeq \frac{L_{\mathrm{w}}}{\mu_{\mathrm{w}} \gamma_{\mathrm{w}} m c^{2}} \max \left(\frac{R}{\gamma_{\mathrm{w}}^{2} c}, t_{\mathrm{FRB}}\right)$.

If the total scattered energy exceeds the total wind energy $\mu_{\mathrm{w}} N_{\mathrm{e}} \gamma_{\mathrm{w}} m c^{2}$, then the wind should be significantly accelerated by the photon momentum, which decreases $E_{\mathrm{rad}}$. Thus, the scattered energy should generally be written as

$E_{\mathrm{sca}}=N_{\mathrm{e}} \min \left(E_{\mathrm{rad}}, \mu_{\mathrm{w}} \gamma_{\mathrm{w}} m c^{2}\right)$.

Therefore, we obtain the ratio between scattered energy and the FRB wave energy:

$\frac{E_{\mathrm{sca}}}{E_{\mathrm{FRB}}} \simeq \frac{L_{\mathrm{w}, 37}}{L_{43}} \min \left(\frac{E_{\mathrm{rad}}}{\mu_{\mathrm{w}} \gamma_{\mathrm{w}} m c^{2}}, 1\right) \max \left(\frac{R_{13}}{3 \gamma_{\mathrm{w}}^{2} t_{\mathrm{FRB},-3}}, 10^{-6}\right)$.

Fig. 2 shows $\log \left(E_{\mathrm{sca}} / E_{\mathrm{FRB}}\right)$ as a function of $L_{\mathrm{w}}$ and $\gamma_{\mathrm{w}}$ for a typical weak burst from FRB 121102. If the FRB source is within the magnetosphere of a neutron star (below the light cylinder), we ruled out the presence of a mildly relativistic $\left(\gamma_{\mathrm{w}} \lesssim 10\right)$ wind from the progenitor star with $L_{\mathrm{w}} \gtrsim 10^{39} \mathrm{erg} \mathrm{s}^{-1}$, regardless of its composition (pair or electron-proton), since the FRB pulse would lose a large fraction of its energy. We note that the persistent radio source associated with FRB 121102 (luminosity $\sim 10^{39} \mathrm{erg} \mathrm{s}^{-1}$, at projected distance of $<40 \mathrm{pc}$; Chatterjee et al. 2017; Marcote et al. 2017) may still be powered by an ultrarelativistic $\gamma_{\mathrm{w}} \gg 10$ wind like that in the Crab Nebula.

\section{PARTICLE ACCELERATION DUE TO INDUCED COMPTON SCATTERINGS}

In this section, we calculate particle acceleration by scattering photons when the occupation numbers of both the initial and the final photon states are very large, i.e. due to IC scatterings. The calculations described here are valid when the wave non-linearity parameter ' $a$ ' (equation 2) is less than 1; IC scattering for highly non-linear waves is a complicated problem and will be taken up in a future paper; however, non-linear effects when $a>1$ are included in numerical calculations presented in Fig. 3.

It is best to view the process from the rest frame of an electron. A photon of wave vector $\mathbf{k}^{\prime}$ is scattered to $\mathbf{k}_{1}^{\prime}$ in electron rest frame. The momentum kick given to the electron in this scattering is $\mathbf{k}^{\prime}-\mathbf{k}_{\mathbf{1}}^{\prime}$. The inverse of the process where $\left(k_{2} \hat{\mathbf{k}}_{1}^{\prime}\right) \rightarrow \mathbf{k}^{\prime}$ gives an almost exact opposite kick to the electron; $\hat{\mathbf{k}}_{1}^{\prime} \equiv \mathbf{k}_{\mathbf{1}}^{\prime} /\left|\mathbf{k}_{\mathbf{1}}^{\prime}\right|$ is a unit vector. The non-zero difference between the two is due to the electron recoil in this scattering process so that $k_{1}^{\prime} \neq k^{\prime}$.

The rate of momentum transfer to an electron by a narrow beam of photons with wave vector within $\mathrm{d}^{3} k^{\prime}$ and its inverse process is

$$
\begin{aligned}
\delta \mathbf{p}^{\prime}= & \frac{c \hbar}{2} \frac{\mathrm{d} \sigma_{\mathrm{T}}}{\mathrm{d} \Omega_{\chi^{\prime}}}\left[\left(1+n_{\mathbf{k}_{1}^{\prime}}\right) n_{\mathbf{k}^{\prime}}\left(\mathbf{k}^{\prime}-\mathbf{k}_{1}^{\prime}\right) \frac{\mathrm{d}^{3} k^{\prime}}{4 \pi^{3}}\right. \\
& \left.+\left(1+n_{\mathbf{k}^{\prime}}\right) n_{\mathbf{k}_{2}}\left(\mathbf{k}_{\mathbf{2}}^{\prime}-\mathbf{k}^{\prime}\right) \frac{\mathrm{d}^{3} k_{2}^{\prime}}{4 \pi^{3}}\right] \mathrm{d} \Omega_{\chi^{\prime}},
\end{aligned}
$$

where

$$
\begin{aligned}
\cos \chi^{\prime} & \equiv \hat{\mathbf{k}}^{\prime} \cdot \hat{\mathbf{k}}_{1}^{\prime}, \quad k_{1}^{\prime}=k^{\prime}-\Delta k^{\prime}, \quad k_{2}^{\prime}=k^{\prime}+\Delta k^{\prime}, \\
\mathrm{d} k_{2}^{\prime} & =\mathrm{d} k^{\prime}\left(1+2 \Delta k^{\prime} / k^{\prime}\right), \quad \text { and } \quad \hbar \Delta k^{\prime}=\frac{\hbar^{2} k^{\prime 2}}{m c}\left(1-\cos \chi^{\prime} \ell^{24}\right)
\end{aligned}
$$

is the momentum recoil suffered by the electron due to scattering of one photon of momentum $\hbar \mathbf{k}^{\prime}$ by an angle $\chi^{\prime}$. The factor $4 \pi^{3}$ in the denominator in equation (23) is because the number of distinct photon quantum states in volume $\mathrm{d}^{3} k^{\prime}$ is $\mathrm{d}^{3} k^{\prime} / 4 \pi^{3}$.

We are considering the case where $n_{\mathbf{k}} \gg 1$ is a function of $|\mathbf{k}|$ within a cone, in the $\mathbf{k}$-space, of opening angle $\theta_{\mathrm{s}} \ll 1$. Expressing $k_{1}^{\prime}$ and $k_{2}^{\prime}$ in terms of $k^{\prime}$ (equation 24), and Taylor expansion of $n_{\mathbf{k}_{1}^{\prime}}$ and $n_{k_{2}^{\prime}}$ in terms of $n_{\mathbf{k}^{\prime}}$ transforms equation (23) to the following expression:

$$
\begin{aligned}
\delta \mathbf{p}^{\prime}= & \frac{\mathrm{d} \sigma_{\mathrm{T}}}{\mathrm{d} \Omega_{\chi^{\prime}}} \frac{\hbar k^{\prime 3} \mathrm{~d} k^{\prime} \mathrm{d} \Omega_{\chi^{\prime}}}{4 \pi^{3}}\left[\left(\hat{\mathbf{k}}^{\prime}-\hat{\mathbf{k}}_{1}^{\prime}\right) \mathrm{d} \Omega_{\mathbf{k}^{\prime}}-\left(\hat{\mathbf{k}}^{\prime}-\hat{\mathbf{k}}_{1}^{\prime}\right) \mathrm{d} \Omega_{\mathbf{k}_{\mathbf{1}}^{\prime}}\right. \\
& +\frac{\Delta k^{\prime}}{k^{\prime}}\left(\hat{\mathbf{k}}_{1}^{\prime} \mathrm{d} \Omega_{\mathbf{k}^{\prime}}+\hat{\mathbf{k}}_{1}^{\prime} \mathrm{d} \Omega_{\mathbf{k}_{1}^{\prime}}\right) \\
& \left.+\frac{2\left(\mathbf{k}_{\mathbf{1}}^{\prime}-\mathbf{k}^{\prime}\right) \Delta k^{\prime}}{k^{\prime}} \frac{\partial\left(n_{\mathbf{k}_{\mathbf{1}}^{\prime}} k_{1}^{\prime 2}\right)}{k_{1}^{\prime} n_{\mathbf{k}_{1}^{\prime}} \partial k_{1}^{\prime}} \mathrm{d} \Omega_{\mathbf{k}_{\mathbf{1}}^{\prime}}\right] c n_{\mathbf{k}^{\prime}} n_{\mathbf{k}_{\mathbf{1}}^{\prime}} .
\end{aligned}
$$

Integrating the above expression over incident and scattered photon directions $\left(\Omega_{\mathbf{k}^{\prime}}\right.$ and $\left.\Omega_{\mathbf{k}_{1}^{\prime}}\right)$ and frequency yields the total rate of momentum deposit to the electron by the IC scatterings. The $d \Omega_{\mathbf{k}^{\prime}}$ and $d \Omega_{\mathbf{k}_{1}^{\prime}}$ integrals, nested inside the square brackets in equation (25), are carried out subject to the condition that the scattering angle $\chi^{\prime}$ is held fixed. Thus, the first two terms in the square brackets cancel exactly when the angular integral over photon propagation direction is performed. The integral of the last term over $\Omega_{\mathbf{k}_{1}^{\prime}}$ is smaller than the third term by at least a factor $\theta_{\mathrm{s}}^{\prime-2}\left(\theta_{\mathrm{s}}^{\prime}\right.$ is half of angular size of the radiation beam in the electron rest frame). This is because both the incident and scattered photons lie within the angle $\theta_{\mathrm{s}}^{\prime}$ for the IC scattering ${ }^{1}$ to be relevant, and therefore, $\left|\mathbf{k}_{1}^{\prime}-\mathbf{k}^{\prime}\right| \lesssim k^{\prime} \theta_{\mathrm{s}}^{\prime 2} / 2$. Thus, the last term in equation (25) can be ignored as well. This simplifies the expression for the radiation force on the electron considerably:

$\frac{\mathrm{d} \mathbf{p}^{\prime}}{\mathrm{d} t^{\prime}} \approx \frac{c \hbar}{2 \pi^{3}} \int \mathrm{d} k^{\prime} k^{\prime 2} \int \mathrm{d} \Omega_{\chi^{\prime}} \frac{\mathrm{d} \sigma_{\mathrm{T}}}{\mathrm{d} \Omega_{\chi^{\prime}}} \int \mathrm{d} \Omega_{\mathbf{k}^{\prime}} \hat{\mathbf{k}}_{1}^{\prime} \Delta k^{\prime} n_{\mathbf{k}^{\prime}} n_{\mathbf{k}_{1}^{\prime}}$.

Since $k^{\prime}-k_{1}^{\prime}=\Delta k^{\prime} \ll k^{\prime}$ (see equation 24), we can take $n_{\mathbf{k}_{1}^{\prime}} \approx$ $n_{\mathbf{k}^{\prime}}$, and the integral over $\Omega_{\mathbf{k}^{\prime}}$ gives $\left(\pi \theta_{\mathrm{s}}^{\prime 2} \Delta k^{\prime} n_{\mathbf{k}^{\prime}}^{2}\right) \hat{\mathbf{z}}$; the axis of the photon beam cone is along $\hat{\mathbf{z}}$, and the photon occupation number

\footnotetext{
${ }^{1}$ Scattering a photon outside the radiation beam $\left(\chi^{\prime} \gtrsim \theta_{\mathrm{s}}^{\prime}\right)$ is a weaker process than the IC scattering within the beam for the FRB parameters being considered here.
} 

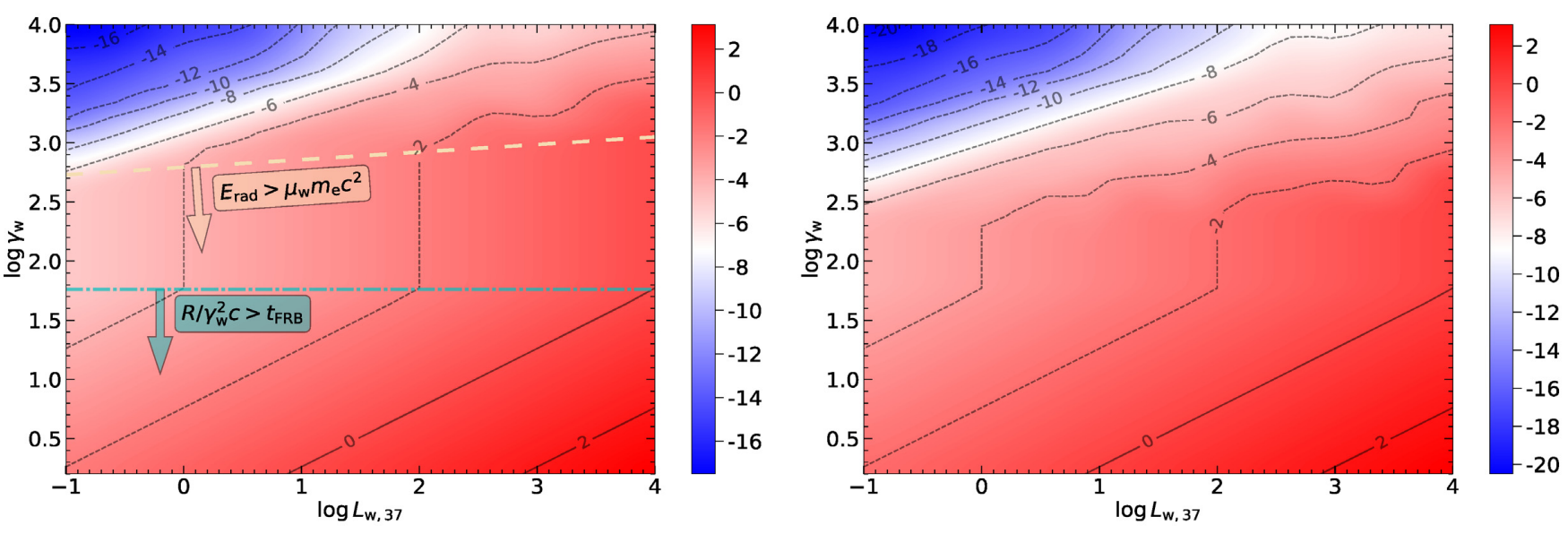

Figure 2. The ratio between scattered energy and FRB energy, $\log \left(E_{\mathrm{sca}} / E_{\mathrm{FRB}}\right)$, as a function of wind power $L_{\mathrm{W}}=10^{37} L_{\mathrm{w}, 37} \mathrm{erg} \mathrm{s}^{-1}$ and $\mathrm{LF} \gamma_{\mathrm{w}}$. The region with $\log \left(E_{\mathrm{sca}} / E_{\mathrm{FRB}}\right)>0$ is ruled out. We consider a typical weak burst from FRB 121102 with $t_{\mathrm{FRB}}=0.1 \mathrm{~ms}$ and $L=10^{41} \mathrm{erg} \mathrm{s}^{-1}$ at $v=1 \mathrm{GHz}$. We assume the source to be located much below the light cylinder of a neutron star $\left(\ll 10^{10} \mathrm{~cm}\right)$ and take the characteristic FRB-wind interaction radius to be $R=10^{10}$ $\mathrm{cm}$. The left-hand panel is for $\mu_{\mathrm{w}}=1$ (corresponding to magnetized $\mathrm{e}^{ \pm}$wind), and the right-hand panel is for $\mu_{\mathrm{w}}=10^{3}$ (magnetized electron-proton wind). The thick dashed and dash-dotted lines in the left-hand panel mark the different regimes of the parameter space (see equation 22). Note that the particle trajectory for $\omega_{\mathrm{B}} \gg \omega$ and $a \gg 1$ is chaotic, and we have carried out convergence tests with different time resolutions to confirm the qualitative results.

$\left(n_{\mathbf{k}^{\prime}}\right)$ is taken to be angle independent inside the radiation cone, i.e. for $\theta^{\prime}<\theta_{\mathrm{s}}^{\prime}$. Thus, equation (26) reduces to

$$
\begin{aligned}
\frac{\mathrm{d} \mathbf{p}^{\prime}}{\mathrm{d} t^{\prime}} \approx & \frac{3 \hbar^{2} \sigma_{\mathrm{T}} \theta_{\mathrm{s}}^{\prime 2}}{4 \pi^{2} m} \int \mathrm{d} k^{\prime} k^{\prime 4} n_{\mathbf{k}^{\prime}}^{2} \int_{0}^{\theta_{\mathrm{s}}^{\prime}} \mathrm{d} \chi^{\prime} \sin \chi^{\prime} \sin ^{2} \Theta^{\prime} \\
& \times \sin ^{2}\left(\chi^{\prime} / 2\right) \hat{\mathbf{z}},
\end{aligned}
$$

where we made use of equation (24) for $\Delta k^{\prime}$ and

$$
\frac{\mathrm{d} \sigma_{\mathrm{T}}}{\mathrm{d} \cos \chi^{\prime}}=\frac{3 \sigma_{\mathrm{T}}}{4} \sin ^{2} \Theta^{\prime}
$$

which is the differential scattering cross-section of an electron for linearly polarized radiation, where $\Theta^{\prime}$ is the angle between the electric vector of incident radiation and the momentum vector of scattered photons in the electron rest frame $\left(\Theta^{\prime} \approx \pi / 2\right.$ for small angle scatterings that are relevant for IC acceleration).

The final two integrals are straightforward and are carried out assuming that $\theta_{\mathrm{s}}^{\prime} \ll 1$ and $n_{\mathbf{k}^{\prime}}$ is a smooth function of $k^{\prime}$ :

$$
\frac{\mathrm{d} \mathbf{p}^{\prime}}{\mathrm{d} t^{\prime}} \approx \frac{3 \hbar^{2} \sigma_{\mathrm{T}} \theta_{\mathrm{s}}^{\prime 6} k^{\prime 5} n_{\mathbf{k}^{\prime}}^{2}}{64 \pi^{2} m} .
$$

We can transform this equation to lab frame by noting that $\mathrm{d} p_{z}^{\prime} / \mathrm{d} t^{\prime}=$ $\mathrm{d} p_{z} / \mathrm{d} t$ since the Lorentz boost is in the same direction as $\delta \mathbf{p}^{\prime}$ and the change to the particle energy in the co-moving frame is proportional to $\left|\delta \mathbf{p}^{\prime}\right|^{2}$. Moreover,

$$
\begin{aligned}
\sin \theta_{\mathrm{s}}^{\prime} & =\frac{\sin \theta_{\mathrm{s}}}{\gamma\left(1-\beta \cos \theta_{\mathrm{s}}\right)} \approx 2 \gamma \theta_{\mathrm{s}}, \quad \text { for } \quad\left(\theta_{\mathrm{s}} \gamma\right) \ll 1, \\
k^{\prime} & =k \gamma\left(1-\beta \cos \theta_{\mathrm{s}}\right) \approx k / 2 \gamma, \quad \text { for } \quad\left(\theta_{\mathrm{s}} \gamma\right) \ll 1,
\end{aligned}
$$

which leads to

$$
\frac{\mathrm{d} p_{z}}{\mathrm{~d} t} \approx \frac{3 \hbar^{2} \sigma_{\mathrm{T}} \theta_{\mathrm{s}}^{6} k^{5} \gamma n_{\mathbf{k}^{\prime}}^{2}}{32 \pi^{2} m}
$$

The photon occupation number, $n_{\mathbf{k}^{\prime}}$, is a Lorentz invariant quantity and it can be easily shown to be

$$
n_{k}=\frac{c^{2} L_{v}}{8 \pi^{2} \theta_{\mathrm{s}}^{2} R^{2} h v^{3}} \sim \frac{c^{2} L}{8 \pi^{2} \theta_{\mathrm{s}}^{2} R^{2} h v^{4}},
$$

where $L_{v}$ is the specific luminosity (isotropic equivalent) and $R$ is distance from the FRB source where particle acceleration is being considered. Substituting this into equation (31), we finally obtain

$$
\frac{\mathrm{d} p_{z}}{\mathrm{~d} t} \approx \frac{3 \sigma_{\mathrm{T}} \theta_{\mathrm{s}}^{2} L^{2} \gamma}{256 \pi^{3} R^{4} v^{3} m c}
$$

If the inertia of the medium is dominated by electrons and positrons, then $p_{z}=m c \beta \gamma$, and the equation for LF of the particle is

$\frac{\mathrm{d} \beta \gamma}{\mathrm{d} t} \approx \frac{3 \sigma_{\mathrm{T}} \theta_{\mathrm{s}}^{2} L^{2} \gamma}{256 \pi^{3} R^{4} \nu^{3} m^{2} c^{2}}$

This result is easy to understand as follows. Since for IC scatterings the scattered photon lies within the photon beam of opening angle $\theta_{\mathrm{s}}^{\prime}$, the scattering cross-section is $\sigma_{\mathrm{T}} \theta_{\mathrm{s}}^{\prime 2} n_{k}$. In each scattering, the electron is recoiled and the momentum impulse it receives - when we subtract the contribution from its inverse process - is $\sim h^{2} v^{\prime 2} \theta_{\mathrm{s}}^{\prime 2} /\left(2 m c^{3}\right)$. The number of photons streaming outward per unit area and time is $L^{\prime} /\left(4 \pi R^{2} h v^{\prime}\right)$. Combining all of these pieces, we find the radiative force on an electron to be $\sigma_{\mathrm{T}} L n_{k} h \nu \theta_{\mathrm{s}}^{4} \gamma /\left(8 \pi R^{2} m c^{3}\right)$, where we made use of Lorentz transformations, i.e. $\theta_{\mathrm{s}}^{\prime} \sim \gamma \theta_{\mathrm{s}}, v^{\prime} \sim v / \gamma$, and $L^{\prime} \sim L / \gamma^{2}$. Substituting for $n_{k}$ from equation (32), we arrive at an expression for the radiative force on the electron that is within a factor 2 of that given in equation (33). It should be noted that particle acceleration is more severe when the medium through which the coherent radiation propagates is moving away from the source at relativistic speeds.

\section{CONSTRAINTS ON FRB RADIATION MECHANISMS}

We consider two cases in separate sections. The first one is where the magnetic field in the source is weak, $B \lesssim 10^{3} \mathrm{G}$, so that the cyclotron frequency is less than $1 \mathrm{GHz}$ and the scattering of FRB radiation is not affected by the magnetic field. The other case is that of a strong magnetic field, which is analysed in Section 4.2. 

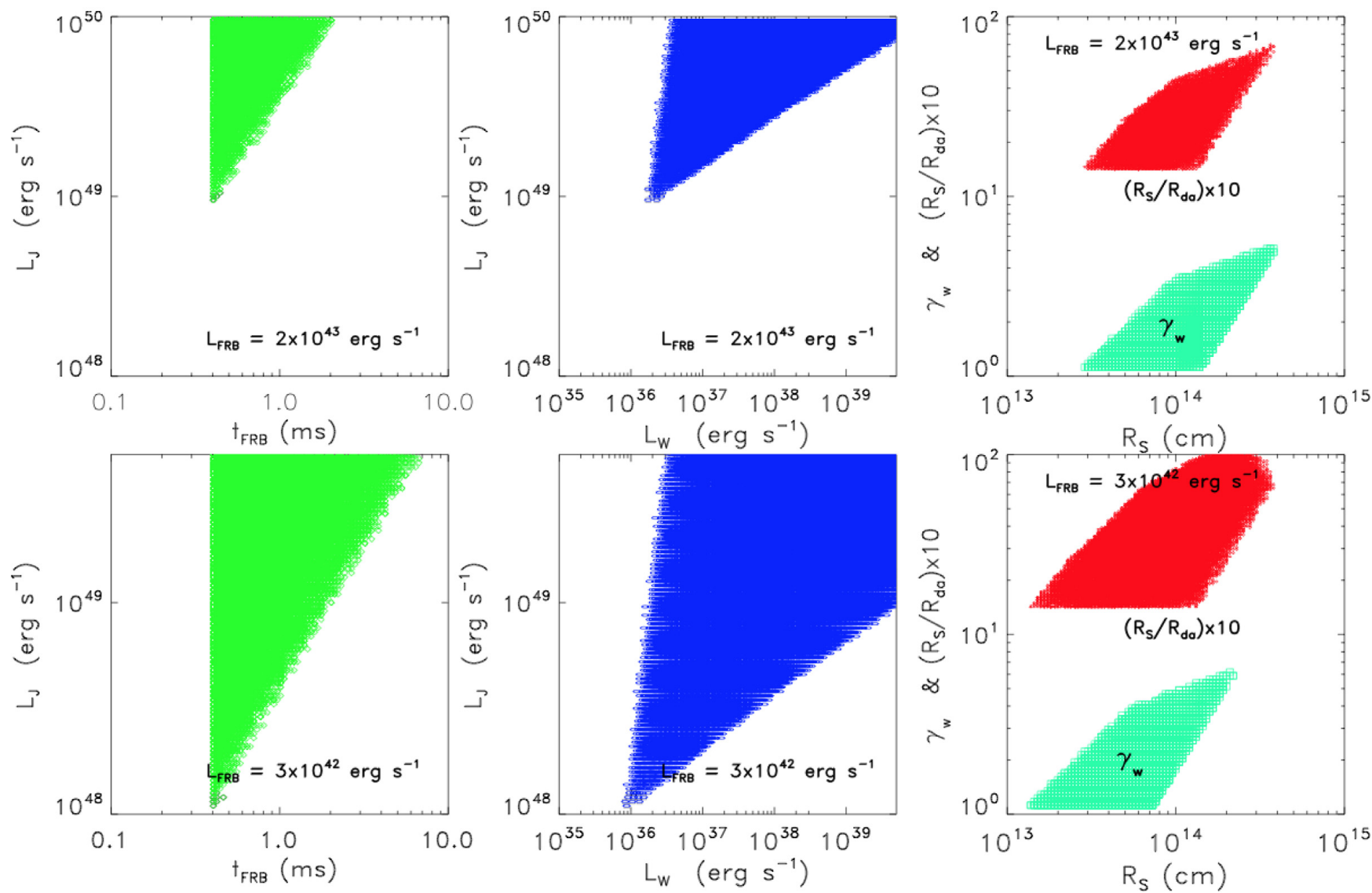

Figure 3. We show results of numerical calculations for FRB emission according to maser mechanisms operating in shocks that result from a relativistic jet of luminosity $L_{\mathrm{j}}$ colliding with a cold wind of luminosity $L_{\mathrm{w}}$, both of which are produced by the same compact object. Each panel shows a pair of parameters that survive the constraint imposed by particle acceleration upstream of the shock front that shuts off the generation of coherent radiation. Constraints are placed on the particle acceleration time due to IC scatterings $-t_{\mathrm{acc}}\left(R_{\mathrm{S}}\right)>t_{\mathrm{FRB}} / 10$, where $t_{\mathrm{FRB}}$ is burst duration in observer frame that is calculated using equation (49), and $t_{\mathrm{acc}}\left(R_{\mathrm{S}}\right)$, given by equation (39), is particle acceleration time due to IC scattering at the shock radius $R_{\mathrm{S}}$ [we note that our numerical calculation of $t_{\mathrm{acc}}$ includes non-linear effects when the wave non-linear parameter $a>1$, whereas equation (39) is valid only for $a \ll 1$ ]. For the purpose of calculating upstream particle accelerations by the IC process, we have taken the observed, isotropic, FRB luminosity to be either $2 \times 10^{43} \mathrm{erg} \mathrm{s}^{-1}$ (the top three panels) - the median luminosity of 'non-repeating FRBs' is $\sim 10^{44} \mathrm{erg} \mathrm{s}^{-1}$ (e.g. Luo et al. 2018; Ravi 2019a,b) - or $3 \times 10^{42} \mathrm{erg} \mathrm{s}^{-1}$ (bottom three panels), which is roughly the median luminosity for the bursts of the repeater FRB 121102 (e.g. Michilli et al. 2018; Hessels et al. 2019). We do not impose a prior on this luminosity as it is dependent on the details of the maser process, whereas the focus of this paper is to provide general constraints that should apply to all maser mechanisms operating in shocks. We emphasize that the luminosity information is used only for the calculation of upstream particle acceleration and nothing else. We show in this figure the parameter space that yields the burst duration $t_{\mathrm{FRB}}>0.4 \mathrm{~ms}$, and the peak frequency $\nu_{\mathrm{B}}$ (given by equation 50) greater than $0.4 \mathrm{GHz}($ which is the lowest frequency at which these bursts have been detected by CHIME; Amiri et al. 2019a, b). The parameter search is cut off at $L_{\mathrm{j}}>10^{50}$ erg s ${ }^{-1}$ and $L_{\mathrm{W}}>5 \times 10^{39} \mathrm{erg} \mathrm{s}^{-1}$. The luminosity for the biggest magnetar flare we have ever observed (SGR 1806-20) was about $2 \times 10^{47} \mathrm{erg} \mathrm{s}^{-1}$ in $\gamma$-rays (Hurley et al. 2005; Palmer et al. 2005), and the energy was smaller in relativistic outflows by a factor of $\sim 10^{2}$ (Gelfand et al. 2005; Granot et al. 2006). The upper limit on $L_{\mathrm{j}}$ of $10^{50} \mathrm{erg} \mathrm{s}^{-1}$ we have set in our numerical calculations is a factor of $\sim 10^{5}$ larger than the luminosity of mildly relativistic outflows for SGR 1806-20. The observed FRB frequency is taken to be the cyclotron frequency $v_{\mathrm{B}}$. What we find is that the maser-in-shock model for FRB radiation requires the isotropic luminosity of the relativistic jet responsible for the FRB radiation to be $\gtrsim 10^{49} \mathrm{erg} \mathrm{s}^{-1}$ (top and bottom left and middle panels), and that means that the total outburst energy of the repeater FRB 121102 in just $1 \mathrm{yr}$ exceeds the magnetic field energy of a magnetar with surface field strength of $\sim 10^{15} \mathrm{G}$; we should point out that the high value of $L_{\mathrm{j}}$ is dictated entirely by the requirement that the emission is produced at a sufficiently large radius $R_{\mathrm{S}}$ and shock LF $\gamma_{\mathrm{sw}}$ (equation 41), so as to avoid excessive particle acceleration upstream of the shock front due to IC, and at the same time produce burst duration $\gtrsim 0.4$ ms; no constraint is placed as to how the maser mechanism operates and its efficiency. The top and bottom left panels show that the jet energy increases very rapidly with increasing burst duration. The top and bottom right panels show that the observed radiation is produced at a radius that is a few times larger than the deceleration radius of the jet, and the wind LF is between $\sim 1$ and 4 for reasons that are explained in Section 4.1.1. The upstream IC optical depth is included in these calculations, but it turns out to be a substantially weaker constraint than IC acceleration. The last point to note is that the maser-in-shock model has no solutions for FRB luminosity $\gtrsim 10^{44} \mathrm{erg} \mathrm{s}^{-1}$; there are solutions if we allow $L_{\mathrm{j}}$ to be larger than $\sim 10^{51} \mathrm{erg} \mathrm{s}^{-1}$, but that poses problems for the total energetics. 
4.1 The medium through which the FRB radiation is passing has weak magnetic field (cyclotron frequency $\lesssim \mathbf{G H z}$ )

The FRB scenario considered in this section is where the coherent radiation is produced when a relativistic jet from a compact object interacts with the CSM and a fraction of the jet energy is converted to $\mathrm{GHz}$ photons. We provide general constraints on the viability of this model.

The ability of the shock model to reproduce the observed duration of FRBs and their frequency can be robustly constrained by combining a few physical considerations. For much of the following discussions, we will ignore factors of order unity.

If the FRB radiation were to be produced at radius $\lesssim 10^{13} \mathrm{~cm}$, the non-linear parameter associated with the radiation at this radius is $a \gtrsim 5$ (equation 2) and electrons upstream of the shock front are accelerated to LF $2 a^{2} \gtrsim 50$ (see Section 2). This high LF of particles upstream completely changes the shock dynamics as well as the cyclotron/synchrotron maser instability growth rate that has been suggested for FRB radiation (Plotnikov \& Sironi 2019). Furthermore, IC scatterings upstream of the shock front provide important constraint on the shock model for FRB radiation.

The IC scattering optical depth ahead of the shock front is given by (e.g. Lyubarsky 2008; Lu \& Kumar 2018)

$\tau_{\mathrm{ic}} \sim \frac{\sigma_{\mathrm{T}} L n_{\mathrm{w}}\left(c t_{\mathrm{FRB}}\right)}{8 \pi^{2} R^{2} m v^{3}} \sim \frac{4 L_{43} n_{\mathrm{w}, 2} t_{\mathrm{FRB},-3}}{R_{13}^{2} v_{9}^{3}}$.

This equation is valid for IC scatterings both within the photon beam and outside the photon beam as long as $R \sim 2 c t_{\mathrm{FRB}} / \theta_{\mathrm{s}}^{2}$, where $\theta_{\mathrm{s}}$ is the beam size of the coherent radiation at $R$ (the shock radius) $\theta_{\mathrm{s}} \approx \gamma_{\mathrm{sw}}^{-1}$ when the radiation is produced in a relativistic shock and the LF of the shocked fluid with respect to the upstream unshocked plasma is $\gamma_{\mathrm{sw}}$. The IC optical depth given in equation (35) is in the CSM rest frame. We need to modify this equation if the upstream CSM is a wind with LF $\gamma_{\mathrm{w}}$. The luminosity in the wind rest frame $L^{\prime} \approx L / \gamma_{\mathrm{w}}^{2}, v^{\prime} \approx \nu / \gamma_{\mathrm{w}}$, and the burst duration in the wind frame is $t_{\mathrm{FRB}}^{\prime} \approx \gamma_{\mathrm{w}}^{2} t_{\mathrm{FRB}}$. Thus, the IC optical depth can be rewritten as

$\tau_{\mathrm{ic}} \sim \frac{\sigma_{\mathrm{T}} L^{\prime} n_{\mathrm{w}}^{\prime}\left(c t_{\mathrm{FRB}}^{\prime}\right)}{8 \pi^{2} R^{2} m v^{\prime 3}} \sim \frac{\sigma_{\mathrm{T}} L L_{\mathrm{w}} \gamma_{\mathrm{w}}\left(c t_{\mathrm{FRB}}\right)}{32 \pi^{3} m^{2} c^{3} \mu_{\mathrm{w}} R^{4} v^{3}}$,

where $L_{\mathrm{w}}$ and $\mu_{\mathrm{w}}$ are wind luminosity and magnetization parameter, respectively, and we used the relation $L_{\mathrm{w}}=4 \pi R^{2} n_{\mathrm{w}}^{\prime} m c^{3} \mu_{\mathrm{w}} \gamma_{\mathrm{w}}^{2}$ to get rid of $n_{\mathrm{w}}^{\prime}$. The requirement that $\tau_{\mathrm{ic}}<1$ provides an upper bound on the CSM density

$n_{\mathrm{w}}(R)=n_{\mathrm{w}}^{\prime} \gamma_{\mathrm{w}} \lesssim\left(30 \mathrm{~cm}^{-3}\right) \frac{R_{13}^{2} \nu_{9}^{3}}{L_{43} t_{\mathrm{FRB},-3} \gamma_{\mathrm{w}}^{2}}$.

For density $n_{\mathrm{w}} \ll 10 \mathrm{~cm}^{-3}$, the relativistic jet deceleration radius and the place where coherent radiation is produced is $\gtrsim 10^{13} \mathrm{~cm}$ (see Section 4.1.1). This alleviates the problem associated with the acceleration of upstream $\mathrm{e}^{ \pm}$away from the shock front to high LF due to the electric field of the coherent FRB radiation. Moreover, at the larger radius, particle acceleration due to IC scatterings also poses less of a problem as we describe next. However, the frequency of maser photons produced in the shock is below the observing band for even CHIME at this low CSM density; this point is discussed further in Section 4.1.1.

The LF of plasma upstream of the shock front - accelerated by IC scatterings of FRB radiation - can be calculated using equation (34), which is rewritten below for the relativistic case in a more convenient form:

$\frac{\mathrm{d} \gamma}{\mathrm{d} t}=\frac{\gamma}{t_{\mathrm{acc}}}$ where $t_{\mathrm{acc}} \sim \frac{256 \pi^{3} R^{4} v^{3} m^{2} c^{2}}{3 \sigma_{\mathrm{T}} \theta_{\mathrm{s}}^{2} L^{2}} \sim\left(3 \times 10^{-13} \mathrm{~s}\right) \frac{R_{13}^{4} v_{9}^{3}}{L_{43}^{2} \theta_{\mathrm{s}}^{2}}$.

This shows that the LF of particles increases exponentially on a very short time-scale, and they attain a terminal LF of $\sim 3 \theta_{\mathrm{s}}^{-1} \approx 3 \gamma_{\mathrm{sw}}$ when the radiation field in the particle co-moving frame becomes nearly isotropic and little momentum is imparted to electrons in further scatterings. If the cold upstream medium moves away from the source with LF $\gamma_{\mathrm{w}}$, then $t_{\mathrm{acc}}$ is smaller by a factor $\gamma_{\mathrm{w}}$ in the lab frame.

The leading front of the radiation that is produced by the shock wave is ahead of the shock by a distance ${ }^{2} \sim \delta R /\left(2 \gamma_{\mathrm{sw}}^{2}\right) \sim$ $c t_{\mathrm{FRB}}(\delta R / R)$, where $\delta R$ is the distance the shock has travelled since the onset of the radiation. Thus, the time available in the lab frame for an upstream particle to be accelerated to $\gamma \sim \gamma_{\text {sw }}$ by the radiation front before it is swept up by the shock is roughly $t_{\mathrm{FRB}} / 5$. This defines a radius, $R_{\mathrm{ra}}$, beyond which radiative acceleration can be ignored. This radius is determined from the condition that $t_{\mathrm{acc}} \sim t_{\mathrm{FRB}} / 10$ :

$R_{\mathrm{ra}} \sim\left(5.5 \times 10^{13} \mathrm{~cm}\right) t_{\mathrm{FRB},-3}^{2 / 5} L_{43}^{2 / 5} v_{9}^{-3 / 5}$,

where we took $\theta_{\mathrm{s}} \sim \gamma_{\mathrm{sw}}^{-1}$, and $\gamma_{\mathrm{sw}}^{2} \sim R_{\mathrm{ra}} /\left(2 c t_{\mathrm{FRB}}\right)$ (see equation 49). We note that $R_{\mathrm{ra}}$ depends extremely weakly on the effective particle inertia (per electron) as $R_{\mathrm{ra}} \propto m_{\mathrm{eff}}^{-1 / 5}$. Therefore, $R_{\mathrm{ra}}$ given by equation (40) does not change by more than a factor of 4 even if the inertia of the upstream plasma is dominated by ions or magnetic field instead of $\mathrm{e}^{ \pm}$. We also note that $R_{\mathrm{ra}}$ is larger by a factor $\gamma_{\mathrm{w}}^{3 / 5}$ when the CSM upstream of the coherent source is moving away with LF $\gamma_{w}$.

If FRB radiation is produced when the shock front is at $R \lesssim R_{\mathrm{ra}} \sim$ $10^{14} \mathrm{~cm}$, then the $\mathrm{e}^{ \pm}$plasma upstream of the shock is accelerated to LF of order $3 \gamma_{\mathrm{sw}}$ due to the IC scattering of the radiation. Since the upstream particle velocity is larger than the shock front speed, and directed away from it, the effect of the IC scatterings is to prevent particles from approaching the front. Thus, radiation produced by the shock for a time much less than $t_{\mathrm{FRB}}$, travelling upstream, accelerates plasma away from it, and halts further production of radiation. IC accelerated particles slow down as they move outward and share their momenta with a larger number of particles. However, even the swept-up medium has relativistic speed up to a distance $\left[\tau_{\text {ic }} \delta E / 4 \pi n m c^{2}\right]^{1 / 3} \sim 5 \times 10^{13} \mathrm{~cm}\left[\tau_{\text {ic }}(\delta E)_{38} / n_{2}\right]^{1 / 3}$ from the shock front, where $\delta E$ is the energy of the radiation pulse produced by the shock before the process is shut off and $\tau_{\text {ic }}$ is the optical depth of the CSM to IC scatterings out to the distance 5 $\times 10^{13} \mathrm{~cm}$ (given by equation 35). We see that just 1 percent of a typical FRB radiation produced at $R \lesssim 10^{14} \mathrm{~cm}$ can drive CMS to speed $\sim c$ and halt the radiation production process.

\subsubsection{Constraining maser mechanisms in shocks for FRB radiation}

Let us consider that the relativistic jet from a compact object interacts with a cold wind with the following properties: the wind is composed of particles of mass $m$, has LF $\gamma_{\mathrm{w}}$, its magnetization

\footnotetext{
${ }^{2}$ This assumes that the wave frequency, $\omega$, is at least a factor few times $\gamma_{\mathrm{sw}}$ larger than the plasma frequency in the upstream medium. This is the situation with many FRB radiation production scenarios, including the cyclotron/synchrotron maser instability mechanism that operates in the shock transition layer and downstream of the shock front. If this condition were not satisfied, then the FRB radiation would not be able to travel away from the shock front to be received by the observer.
} 
parameter (the ratio of magnetic to particle kinetic energy densities) is $\mu_{\mathrm{w}}$, energy density in wind co-moving frame is $u_{\mathrm{w}}^{\prime}$, and its luminosity in the lab frame is $L_{\mathrm{w}} \approx 4 \pi R^{2} u_{\mathrm{w}}^{\prime} \gamma_{\mathrm{w}}^{2} c$. Similarly, the parameters of the relativistic jet are $\gamma_{\mathrm{j}}, \mu_{\mathrm{j}}, u_{\mathrm{j}}^{\prime}$, and $L_{\mathrm{j}}$. Let us take the LF of the shocked wind plasma with respect to the unshocked wind to be $\gamma_{\mathrm{sw}}$, and the shocked jet plasma LF with respect to the unshocked jet fluid to be $\gamma_{\mathrm{sj}}$. It can be shown using the continuity of mass, momentum, and energy flux across the shock front that for a relativistic, magnetized plasma, the energy density of the shocked wind (in shocked fluid rest frame) is $u_{\mathrm{s} \text {,wind }}^{\prime} \approx \gamma_{\mathrm{sw}}^{2} u_{\mathrm{w}}^{\prime}$, and similarly $u_{\mathrm{s}, \mathrm{jet}} \approx \gamma_{\mathrm{sj}}^{2} u_{\mathrm{j}}^{\prime}$ (e.g. Kennel \& Coroniti 1984). The pressure equilibrium between the two shocked fluids, which are separated by the contact discontinuity surface, for highly relativistic systems, requires $u_{\mathrm{s} \text {,jet }}^{\prime}=u_{\mathrm{s} \text {, wind }}^{\prime}$. Moreover, the LF of the jet with respect to the wind, $\sim \gamma_{\mathrm{j}} / \gamma_{\mathrm{w}}$, should be equal to $\sim \gamma_{\mathrm{sj}} \gamma_{\mathrm{sw}}$; i.e. two different ways of calculating the relative speed of the jet and the wind should agree. Combining these two relations, we find

$\gamma_{\mathrm{sw}}^{4} \approx \frac{\gamma_{\mathrm{j}}^{2} u_{\mathrm{j}}^{\prime}}{\gamma_{\mathrm{w}}^{2} u_{\mathrm{w}}^{\prime}} \quad$ or $\quad \gamma_{\mathrm{sw}} \approx\left[\frac{L_{\mathrm{j}}}{L_{\mathrm{w}}}\right]^{1 / 4}$.

The deceleration radius of the jet is the distance it travels before half of its energy is transferred to the CSM. The total energy of the shocked wind, in the lab frame, is $\sim 4 \pi R^{3} u_{\mathrm{w}}^{\prime} \gamma_{\mathrm{sw}}^{2}$. Thus, the deceleration radius is

$E_{\mathrm{j}} \equiv L_{\mathrm{j}} t_{\mathrm{jet}} \approx 8 \pi R_{\mathrm{da}}^{3} u_{\mathrm{w}}^{\prime} \gamma_{\mathrm{sw}}^{2} \Rightarrow R_{\mathrm{da}} \approx \frac{c E_{\mathrm{j}} \gamma_{\mathrm{w}}^{2}}{2 L_{\mathrm{w}} \gamma_{\mathrm{sw}}^{2}}$

where $t_{\text {jet }}$ is the time duration in the lab frame over which the relativistic jet was launched from radius $R_{\text {jet }}$ in the magnetosphere of the magnetar. The shock LF after the deceleration radius is given by the energy conservation equation (42):

$\gamma_{\mathrm{sw}}\left(R>R_{\mathrm{da}}\right) \approx \gamma_{\mathrm{sw}}\left(R_{\mathrm{da}}\right)\left[\frac{R}{R_{\mathrm{da}}}\right]^{1 / 2}$.

The FRB lasts for a time duration of the order of the jet deceleration time in the observer frame, $t_{\mathrm{da}}^{\mathrm{obs}}$, when roughly 50 per cent of the coherent radiation is generated. The observer frame deceleration time can be calculated from $R_{\mathrm{da}}$ and LF of the shock front in the lab frame, ${ }^{3} \gamma_{\mathrm{sw}} \mu_{\mathrm{w}}^{1 / 2} \gamma_{\mathrm{w}}$, using the standard formula for a source that is moving towards the observer with relativistic speed (e.g. Kumar \& Zhang 2015):

$t_{\mathrm{da}}^{\mathrm{obs}} \approx \frac{R_{\mathrm{da}}}{2 c\left[\gamma_{\mathrm{sw}} \gamma_{\mathrm{w}} \mu_{\mathrm{w}}^{1 / 2}\right]^{2}} \approx \frac{t_{\mathrm{jet}}}{4 \mu_{\mathrm{w}}}$,

where we used equations (41) and (42) to arrive at the second equality in (44). The jet launching time, $t_{\text {jet }}$, ought to be smaller than a few ms because of FRB energetics. ${ }^{4}$ Thus, the shock model gives burst duration (equation 44) much smaller than the observed few ms width of FRBs if the magnetization parameter of the wind

\footnotetext{
${ }^{3}$ The LF of the shock front is larger than the LF of the shocked plasma $\left(\gamma_{\mathrm{sw}}\right)$ by a factor $\sim \mu_{\mathrm{w}}^{1 / 2}$ (e.g. Kennel \& Coroniti 1984).

${ }^{4}$ The total energy of the relativistic jet (isotropic equivalent) for a typical FRB is of the order of $10^{42}$ erg if the radiation production efficiency is a few percent. Considering the ms duration of FRBs, the jet energy has to come from the magnetic field and not the neutron star rotation; the rate of energy extraction for the latter is set by the dipole radiation formula and is far smaller than what we see for FRBs (see equation 51). For a magnetar with surface magnetic field of $10^{15} \mathrm{G}$, the total magnetic energy above the radius $R$ is $2 \times 10^{42} R_{8}^{-3} \mathrm{erg}$. Therefore, the jet has be launched at a radius no larger than $\sim 10^{8} \mathrm{~cm}$, i.e. $R_{\text {jet }} \lesssim 10^{8} \mathrm{~cm}$ and $t_{\text {jet }} \approx R_{\text {jet }} / c \lesssim 3 \mathrm{~ms}$.
}

into which the relativistic jet is running into $\left(\mu_{\mathrm{w}}\right)$ is larger than order unity. The possibility that the FRB radiation might be produced at a radius much larger than $R_{\mathrm{da}}$ will be considered later in this section; the constraint on $\mu_{\mathrm{w}}$ when $R \gg R_{\mathrm{da}}$ turns out to be similar. It should also be noted that upstream particles are accelerated by IC scatterings more rapidly and out to larger distances when $\gamma_{\mathrm{w}} \gg 1$ as discussed in Section 4.1, and that is an additional constraint on $\gamma_{\mathrm{w}}$ and $\mu_{\mathrm{w}}$.

Substituting for $\gamma_{\mathrm{sw}}$ from equation (44) into equation (42), and expanding $L_{\mathrm{w}} \approx 4 \pi R_{\mathrm{da}}^{2} \mu_{\mathrm{w}} m c^{3}\left\langle n_{\mathrm{w}}^{\prime}\right\rangle \gamma_{\mathrm{w}}^{2} / 3$, we find

$R_{\mathrm{da}} \sim\left[\frac{3 E_{\mathrm{j}} t_{\mathrm{FRB}} \gamma_{w}^{2}}{4 \pi m c\left\langle n_{\mathrm{w}}^{\prime}\right\rangle}\right]^{1 / 4} \sim\left(5 \times 10^{12} \mathrm{~cm}\right) \gamma_{\mathrm{w}}^{1 / 2}\left[\frac{E_{42} t_{\mathrm{FRB},-3}}{\left\langle n_{\mathrm{w}}^{\prime}\right\rangle_{4}}\right]^{1 / 4}$,

where $\left\langle n_{\mathrm{w}}^{\prime}\right\rangle$ is the mean CSM particle number density in its rest frame within $R_{\mathrm{da}} ;\left\langle n_{\mathrm{w}}^{\prime}\right\rangle=3 n_{\mathrm{w}}^{\prime}\left(R_{\mathrm{da}}\right)$ for a steady wind CSM. This expression is independent of the LF of the relativistic jet and the magnetization of the wind. The second part of equation (45) is obtained by taking the average particle mass in the wind to be the electron mass; $R_{\mathrm{da}}$ is smaller for an ionic wind by a factor of 7 . Considering the weak dependence of $R_{\mathrm{da}}$ on burst energy and CSM density, it is hard for the FRB source, according to the shock model scenario, to be at a radius very different from $10^{13} \mathrm{~cm}$. However, upstream particle acceleration by the emergent radiation renders the shock model not viable at $R \lesssim 10^{13} \mathrm{~cm}$.

As argued earlier, the magnetization parameter of the wind CSM cannot be larger than order unity, otherwise the FRB duration, according to the shock model, would be much smaller than the observed duration. Thus, we take $\mu_{\mathrm{w}} \sim 1$. Moreover, the asymptotic LF of a magnetized wind satisfies the relation $\gamma_{\mathrm{w}} \sim \mu_{\mathrm{w}}^{1 / 2}$ (Goldreich \& Julian 1969; Granot, Komissarov \& Spitkovsky 2011). So, we take $\gamma_{\mathrm{w}}$ to be of order unity as well. Thus, the expected CSM particle density at $R_{\mathrm{da}} \sim 10^{13} \mathrm{~cm}$, for an electron-positron wind, is

$n_{\mathrm{w}} \sim \frac{L_{\mathrm{w}}}{4 \pi R^{2} m c^{3}} \sim\left(3 \times 10^{5} \mathrm{~cm}^{-3}\right) L_{\mathrm{w}, 37} R_{13}^{-2}$,

where our choice of wind luminosity of $10^{37} \mathrm{erg} \mathrm{s}^{-1}$ is for a young magnetar (see equation 51). The high particle density upstream of the shock front makes the CSM highly opaque to IC scattering (equation 35), and that is another problem with the shock model for FRB radiation.

FRB radiation could be generated in shocks at $R \gtrsim 10^{14} \mathrm{~cm}$, where upstream particles are not forced by the radiation to move at relativistic speed away from the shock front. However, if we demand that $R \sim R_{\mathrm{da}}$, so that the FRB radiation is produced efficiently, then that requires the energy of the relativistic jet to be $\gtrsim 10^{46}$ erg or $n$ $\lesssim 1 \mathrm{~cm}^{-3}$ (see equation 45 ). The large energy requirement seems problematic especially for the repeater FRB 121102; the total energy of outbursts in the last $10 \mathrm{yr}$ for this object exceeds the available energy in magnetic fields of even an extreme magnetar if each outburst has $\gtrsim 10^{46}$ erg energy. On the other hand, if we take the CSM density to be sufficiently small so that $R_{\mathrm{da}} \gtrsim 10^{14} \mathrm{~cm}$, then the frequency of coherent radio emission produced in shocks is much smaller than the observed frequencies for FRBs (this point is amplified below).

We next explore the possibility that the FRB radiation is generated at a radius $R_{\mathrm{s}} \gtrsim 10^{14} \mathrm{~cm}$, and relax the efficiency considerations so that $R_{\mathrm{S}}$ can be much larger than the deceleration radius $\left(R_{\mathrm{da}}\right)$ of the relativistic jet.

The characteristic photon frequency for many maser instabilities in plasmas is of the order of the plasma or cyclotron frequency depending on the nature of the instability (the latter is $\mu_{\mathrm{w}}^{1 / 2}$ times the 
former; $\mu_{\mathrm{w}} \equiv B^{2} /\left(8 \pi n_{\mathrm{w}} m c^{2}\right)$ is plasma magnetization parameter). The cyclotron frequency in the shocked wind frame is

$v_{\mathrm{B}}^{\prime} \approx \frac{q B_{\mathrm{w}}^{\prime}}{2 \pi m c}$,

and in the observer frame it is

$\nu_{\mathrm{B}} \approx v_{\mathrm{B}}^{\prime} \gamma_{\mathrm{sw}} \gamma_{\mathrm{w}} \sim \frac{q L_{\mathrm{w}}^{1 / 2} \gamma_{\mathrm{sw}}}{2 \pi m R_{\mathrm{s}} c^{3 / 2}}$

We can eliminate $\gamma_{\mathrm{sw}}$ using the FRB duration for the shock model, namely

$t_{\mathrm{FRB}} \approx \frac{R_{\mathrm{s}}}{2 c\left(\gamma_{\mathrm{sw}} \gamma_{\mathrm{w}} \mu_{\mathrm{w}}^{1 / 2}\right)^{2}}$,

where the factor $\mu_{\mathrm{w}}$ in the denominator is because the shock front LF with respect to the unshocked wind is $\gamma_{\mathrm{sw}} \mu_{\mathrm{w}}^{1 / 2}$ (the LF of shocked plasma with respect to the unshocked wind is $\left.\gamma_{\mathrm{sw}}\right)$. Thus, we arrive at the following expression for the cyclotron frequency of the shocked wind in the observer frame (which should be of the order of the FRB frequency for any maser mechanism apart for a possible factor $\sim \mu_{\mathrm{w}}^{1 / 2}$ ):

$\nu_{\mathrm{B}} \approx \frac{q L_{\mathrm{w}}^{1 / 2} t_{\mathrm{FRB}}^{-1 / 2}}{2^{3 / 2} \pi m c^{2} R_{\mathrm{s}}^{1 / 2} \gamma_{\mathrm{w}} \mu_{\mathrm{w}}^{1 / 2}} \approx\left(7 \times 10^{8} \mathrm{~Hz}\right) \frac{L_{\mathrm{w}, 37}^{1 / 2} t_{\mathrm{FRB},-3}^{-1 / 2}}{R_{\mathrm{s}, 14}^{1 / 2} \gamma_{\mathrm{w}} \mu_{\mathrm{w}}^{1 / 2}}$.

This frequency is at the lower end of the radio band at which FRBs are observed. We see from the above equation that there is little room for $\gamma_{\mathrm{w}} \mu_{\mathrm{w}}^{1 / 2}$ to be much larger than order unity unless $L_{\mathrm{w}} \gg$ $10^{37} \mathrm{erg} \mathrm{s}^{-1}$.

The dipole wind luminosity for a 30-yr-old magnetar with surface field strength of $10^{15} \mathrm{G}$ is (e.g. Goldreich \& Julian 1969)

$L_{\mathrm{W}} \sim 10^{37} \mathrm{erg} \mathrm{s}^{-1} B_{\mathrm{ns}, 15}^{-2} t_{9}^{-2}$,

as long as $t$ is larger than the spin-down time $t_{\mathrm{sd}}$, which is given by

$t_{\mathrm{sd}} \approx 500 \mathrm{~s} B_{\mathrm{ns}, 15}^{-2} P_{-3}^{2}$,

where $P_{-3}$ is the pulsar rotation period in unit of $10^{-3} \mathrm{~s}$. The luminosity is roughly constant for $t \lesssim t_{\mathrm{sd}}$.

The maximum dipole wind luminosity at time $t$ after the birth of an neutron star (NS) is

$L_{\mathrm{w}}^{\max } \sim 10^{39} \mathrm{erg} \mathrm{s}^{-1} P_{-1}^{-2} t_{9}^{-1}$,

which corresponds to $t_{\mathrm{sd}} \sim t$, and the surface magnetic field of

$B \sim\left(7 \times 10^{13} \mathrm{G}\right) P_{-1} t_{9}^{-1 / 2}$,

where $P_{-1}$ is the pulsar rotation period in unit of $0.1 \mathrm{~s}$ at time $t$.

It is entirely possible that the pulsar magnetic field, especially for a young system, is highly non-dipolar. Moreover, the magnetar wind might not be rotationally powered, but instead launched by magnetic field dissipation. For these cases, the wind luminosity provided by equations (51)-(53) does not apply, and for that reason we consider $L_{\mathrm{w}}$ as high as $10^{40} \mathrm{erg} \mathrm{s}^{-1}$ in all of our numerical calculations presented in Fig. 3.

The electron density associated with the magnetar $\mathrm{e}^{ \pm}$wind at $R_{\mathrm{S}}$ is $n_{\mathrm{w}} \sim L_{\mathrm{w}} /\left(4 \pi R_{\mathrm{s}}^{2} m c^{3} \gamma_{\mathrm{w}} \mu_{\mathrm{w}}\right) \sim 3 \times 10^{3} \mathrm{~cm}^{-3} L_{\mathrm{w}, 37} R_{\mathrm{s}, 14}^{-2}\left(\gamma_{\mathrm{w}} \mu_{\mathrm{w}}\right)^{-1}$. The density marginally exceeds the upper limit given in equation (37) - to avoid the medium upstream of the shock front to become opaque to IC scatterings - unless $\gamma_{\mathrm{w}} \mu_{\mathrm{w}} \gg 1$.

The LF of the shock at $R_{\mathrm{s}} \gtrsim 10^{13} \mathrm{~cm}$ should be $\gtrsim 10^{3} \gamma_{\mathrm{w}}^{-1} \mu_{\mathrm{w}}^{-1 / 2}$ in order to produce an ms duration burst (equation 49). This requires the luminosity of the relativistic jet, obtained from equation (41), to be $L_{\mathrm{j}} \sim L_{\mathrm{w}} \gamma_{\mathrm{sw}}^{4} \gtrsim 10^{49} \gamma_{\mathrm{w}}^{-4} \mu_{\mathrm{w}}^{-2} L_{\mathrm{w}, 37} \mathrm{erg} \mathrm{s}^{-1}$.
The allowed parameter space for the maser-in-shock model of FRB is shown in Fig. 3. The parameters are for an FRB with observed luminosity of $2 \times 10^{43} \mathrm{erg} \mathrm{s}^{-1}$ (top three panels), which corresponds to the low end of the luminosity of non-repeaters (e.g. Luo et al. 2018; Ravi 2019a,b). The lower three panels of Fig. 3 show results for radio luminosity of $3 \times 10^{42} \mathrm{erg} \mathrm{s}^{-1}$, which is the median luminosity for bursts of the repeater FRB 121102 (e.g. Michilli et al. 2018; Hessels et al. 2019). The observed luminosity is used for the calculation of particle acceleration upstream of the shock front, and for no other aspect of the maser mechanism. As the left top and bottom panels of the figure show, the energy requirement for the maser-in-shock model grows very rapidly with increasing $t_{\mathrm{FRB}}$. For bursts of duration longer than $\sim 2 \mathrm{~ms}, L_{\mathrm{j}} \gtrsim 10^{49} \mathrm{erg}$ $\mathrm{s}^{-1}$. The energy requirement is reduced if the FRB frequency is much larger than the cyclotron frequency $v_{\mathrm{B}}$ considered in these calculations.

There are no solutions when the relativistic jet luminosity $L_{\mathrm{j}}$ $\lesssim 10^{49} \mathrm{erg} \mathrm{s}^{-1}$ (top left and middle panels of Fig. 3), and it is a serious problem for the maser-in-shock model for FRBs. The wellstudied repeater FRB 121102 has been observed for about $10 \mathrm{yr}$, and $\gtrsim 10^{2}$ outbursts have been detected during the small observing time invested to follow this object. The object has had numerous outbursts with radio luminosity in the $\mathrm{GHz}$ band of $\gtrsim 10^{43} \mathrm{erg} \mathrm{s}^{-1}$. Each of these bursts require, according to the maser model, $L_{\mathrm{j}} \gtrsim$ $10^{49} \mathrm{erg} \mathrm{s}^{-1}$, and therefore, the total energy needed for outbursts in a year is at least $10^{48} \mathrm{erg}$ if the efficiency for converting the magnetic energy to relativistic outflows is 100 per cent. We know empirically that giant magnetar outbursts convert less than a few percent of magnetic energy to mildly relativistic outflows, ${ }^{5}$ and perhaps a much smaller fraction to ultrarelativistic jet that is invoked by maser-inshock models for FRB radio emission. This greatly exacerbates the energy problem - the energy requirement for the maser-in-shock model to support the activities of the FRB repeater for $1 \mathrm{yr}$ is $\gtrsim 10^{50}$ erg and that is hard for magnetic fields, even those as large as $10^{16}$ $\mathrm{G}$, to provide. We note that the value of $L_{\mathrm{j}}$ in our calculations is dictated solely by the shock LF $\gamma_{\mathrm{sw}}$ and $R_{\mathrm{s}}$ such that the observed duration of bursts comes out to be of the order of a few ms, and not by any efficiency considerations.

The maser model yields no solution for FRB luminosity $\gtrsim 10^{44}$ $\mathrm{erg} \mathrm{s}^{-1}$ when the acceleration of upstream particles by IC scatterings shuts off the maser mechanism in less than $1 \mathrm{~ms}$ unless we consider the luminosity of the relativistic jet $L_{\mathrm{j}} \gtrsim 10^{51} \mathrm{erg} \mathrm{s}^{-1} ; L_{\mathrm{j}}$ scales linearly with the FRB radio luminosity. We note that the analysis of ASKAP sample of bursts shows that there are FRBs with luminosity as high as $\sim 10^{46} \mathrm{erg} \mathrm{s}^{-1}$ (Lu \& Piro 2019).

The main result of this section is that it is highly unlikely that the FRB radiation is produced at a distance much larger than a few hundred neutron star radii. Particles in the region $10^{9} \mathrm{~cm} \lesssim$ $R_{\mathrm{S}} \lesssim 10^{13} \mathrm{~cm}$ suffer very strong radiative acceleration that disrupts the photon generation process. The energy requirement for maserin-shock models operating at $R_{\mathrm{S}} \gtrsim 10^{13} \mathrm{~cm}$ is very challenging $\left(\gtrsim 10^{48}\right.$ erg in ultrarelativistic outflows in $1 \mathrm{yr}$ for repeaters such as FRB 121102). For $R \lesssim 10^{9} \mathrm{~cm}$, the strong magnetic field of a

\footnotetext{
${ }^{5}$ The giant magnetar outburst of 2004 December 27 (SGR 1806-20) released $4 \times 10^{46} \mathrm{erg}$ in $\gamma$-rays (Hurley et al. 2005; Palmer et al. 2005), whereas the energy in mildly relativistic outflow, from radio observations, was estimated to be a few times $10^{44} \mathrm{erg}$ (Gaensler et al. 2005; Gelfand et al. 2005; Granot et al. 2006). The peak luminosity for this burst, which was larger by a factor of $\sim 10^{2}$ compared with the previous two most luminous SGRs, was $\sim 2 \times 10^{47} \mathrm{erg} \mathrm{s}^{-1}$. The duration of the initial $\gamma$-ray spike, which carried most of the energy release, for all three giant SGR flares was $0.2 \mathrm{~s}$.
} 
magnetar suppresses the IC scatterings and acceleration of particles by the electric field of FRB coherent radiation (discussed in the next section). Therefore, the generation of coherent photons can proceed unimpeded close to the neutron star.

Although almost all the discussions in this section have explicitly considered the scenario where FRB radiation is generated in shocks, the same physical considerations - acceleration of particles to high LF by the emergent radiation in the vicinity of the source region - apply to any other model for FRBs such as plasma maser-type mechanism that operate far away from the neutron star surface and outside the light cylinder.

\subsection{FRB radiation generation and propagation in a region of strong magnetic field}

Photon-electron scattering cross-section is significantly modified in the presence of strong magnetic fields when the cyclotron frequency $v_{\mathrm{B}} \gtrsim v$. The cross-section for an X-mode photon ${ }^{6}$ is (e.g. Canuto, Lodenquai \& Ruderman 1971)

$\sigma_{\mathrm{x}}=\frac{\sigma_{\mathrm{T}}}{2}\left[\frac{v^{2}}{\left(v+v_{\mathrm{B}}\right)^{2}}+\frac{v^{2}}{\left(v-v_{\mathrm{B}}\right)^{2}}\right]$,

and the cross-section when the wave electric field is not perpendicular to the static magnetic field is

$\sigma_{\|}=\sigma_{\mathrm{T}}\left[\sin ^{2} \theta_{\mathrm{kB}}+\frac{\cos ^{2} \theta_{\mathrm{kB}}}{2}\left\{\frac{v^{2}}{\left(v+v_{\mathrm{B}}\right)^{2}}+\frac{v^{2}}{\left(v-v_{\mathrm{B}}\right)^{2}}\right\}\right]$,

where the cyclotron frequency

$v_{\mathrm{B}}=\frac{q B}{2 \pi m c}$,

$\sin \theta_{\mathrm{kB}} \approx \theta_{\mathrm{kB}}$ is the dot product of unit vectors along the static magnetic field and wave electric field as measured in the electron rest frame, and $v$ is EM wave frequency also in the electron rest frame. These formulae for the cross-section apply only when the EM wave non-linearity parameter $a_{\|} \equiv q E_{\|} / m c \omega$ (see equation 2) is much less than $1 ; E_{\|}$is the component of wave electric vector amplitude along the static magnetic field. For the X-mode we are considering here, the angle between the wave electric field and the magnetic field is very close to $\pi / 2$ (equation 60 ), and $a_{\|} \ll 1$; this point is further addressed later (60).

We see from equation (39) that for $R \lesssim 10^{13} \mathrm{~cm}$ the time-scale for particle acceleration due to IC scatterings is extremely short, provided that the cross-section for scattering a photon by an electron into an unoccupied state is not drastically smaller than $\sigma_{\mathrm{T}}$. However, as we see from equation (55), $\sigma_{\mathrm{x}}$ is smaller than $\sigma_{\mathrm{T}}$ by a factor $\left(v_{\mathrm{B}} / v\right)^{2}$ in the region of high magnetic field. Attenuation of Omodes $^{7}$ by interactions with electrons in the medium in the vicinity of the source is also suppressed by a factor $\sim \theta_{\mathrm{kB}}^{-2}$; modes with $\theta_{\mathrm{kB}} \ll 1$ are unlikely to be able to escape the immediate vicinity of the source region intact.

Consider an $\mathrm{X}$-mode generated at radius $R_{\mathrm{S}}$ (measured from the centre of the host neutron star with strong magnetic field) such that $R_{\mathrm{s}} \lesssim 10^{7} \mathrm{~cm}$. It was shown by Lu, Kumar \& Narayan (2019) that the electric vector of an X-mode travelling through a medium with non-uniform magnetic field rotates in such a way as to keep

\footnotetext{
${ }^{6} \mathrm{An} \mathrm{X}$-mode is a linearly polarized EM wave with the direction of the wave electric field vector perpendicular to the plane of static magnetic field and the wave vector.

${ }^{7} \mathrm{An}$ O-mode is a linearly polarized EM wave with the direction of the wave electric field vector in the plane of static magnetic field and the wave vector.
}

the wave electric field pointing nearly perpendicular to the local magnetic field and the wave vector as long as the plasma density is sufficiently large; the index of refraction of the medium for this mode is very close to unity, so the wave vector does not rotate. The rms angle $\left|\hat{\mathbf{E}}_{\mathrm{w}} \times \hat{\mathbf{B}}\right|$ as the wave travels away from the source region is given by ( $\mathrm{Lu}$ et al. 2019)

$\theta_{\mathrm{kB}} \approx \frac{2 \pi c v}{R_{\mathrm{B}} \omega_{\mathrm{p}}^{2}}$,

where $\omega_{\mathrm{p}}^{2}=4 \pi q^{2} n / m$ is the plasma frequency, $n$ is the electron density, and $R_{\mathrm{B}}$ is the radius of curvature of magnetic field lines at the current location of the wave at $R ; R_{\mathrm{B}} \sim R / \theta$ at polar coordinate $(R, \theta)$ with respect to the magnetic axis. Let us take the $\mathrm{e}^{ \pm}$density at $R$ to be $\mathcal{M}$ times the Goldreich-Julian density (Goldreich \& Julian 1969):

$$
\begin{aligned}
n & =\mathcal{M} n_{\mathrm{GJ}}=\frac{\mathcal{M} \mathbf{B} \cdot \Omega_{\mathrm{ns}}}{2 \pi q c} \approx \frac{\mathcal{M} B_{\mathrm{ns}} \Omega_{\mathrm{ns}}}{2 \pi q c}\left(\frac{R_{\mathrm{ns}}}{R}\right)^{3} \\
& \approx 10^{13} \mathrm{~cm}^{-3} \mathcal{M} B_{\mathrm{ns}, 15} \Omega_{\mathrm{ns}}\left(\frac{R_{\mathrm{ns}}}{R}\right)^{3},
\end{aligned}
$$

where $\Omega_{\mathrm{ns}}$ is the angular velocity of the NS.

Substituting this into (58), we find

$\theta_{\mathrm{kB}} \sim 6 \times 10^{-11} \mathcal{M}^{-1} \nu_{9} R_{\mathrm{B}, 8}^{-1} B_{\mathrm{ns}, 15}^{-1} \Omega_{\mathrm{ns}}^{-1}\left(R / R_{\mathrm{ns}}\right)^{3}$,

inside the freeze-out radius given by equation (61). The wave nonlinearity parameter along the magnetic field $a_{\|} \equiv q E_{\|} /(m c \omega)=$ $q E \theta_{\mathrm{kB}} /(m c \omega) \ll 1$ even out to $R / R_{\mathrm{ns}} \sim 10^{3}$ or $R \sim 10^{9} \mathrm{~cm}$, if $\mathcal{M} \sim$ $10^{3}\left(R_{\mathrm{B}} \sim R / \theta \sim 10^{2} R\right.$ in the magnetic polar cap region). Therefore, it is appropriate to use equation (56) for X-mode scattering crosssection even for bright FRBs.

The rotation of wave electric field ceases at a radius, called the freeze-out radius, where the plasma density becomes too small to be able to provide the current needed to rotate the wave electric vector. This occurs at a radius where (Lu et al. 2019)

$\frac{\omega_{\mathrm{p}}^{2}}{\omega^{2}} \sim \frac{a c}{\omega R_{\mathrm{B}}} \Rightarrow \frac{R_{\mathrm{fo}}}{R_{\mathrm{ns}}} \sim 2 \frac{\left(\mathcal{M} B_{\mathrm{ns}, 15} \Omega_{\mathrm{ns}}\right)^{1 / 2}}{L_{43}^{-1 / 4}}\left[\frac{R_{\mathrm{B}}}{R_{\mathrm{ns}}}\right]^{1 / 2}$.

$R_{\mathrm{B}}$ in this equation is calculated at $R_{\mathrm{fo}}$. The FRB radiation might be produced along open magnetic field lines in the polar cap region that has an angular size of $\theta_{\mathrm{pc}}=\left[\Omega_{\mathrm{ns}} R_{\mathrm{ns}} / c\right]^{1 / 2} \sim 5.8 \times 10^{-3}\left(R_{\mathrm{ns}, 6} \Omega_{\mathrm{ns}}\right)^{1 / 2}$ rad. Taking $\theta \sim \theta_{\mathrm{pc}}$ and substituting $R_{\mathrm{B}} \sim R_{\mathrm{fo}} / \theta$ in equation (61) leads to

$\frac{R_{\mathrm{fo}}}{R_{\mathrm{ns}}} \sim 400 \mathcal{M} B_{\mathrm{ns}, 15}\left(\Omega_{\mathrm{ns}} / R_{\mathrm{ns}, 6}\right)^{1 / 2} L_{43}^{-1 / 2}$.

The angle $\theta_{\mathrm{kB}}$ at the freeze-out radius is

$\theta_{\mathrm{kB}}\left(R_{\mathrm{fo}}\right) \sim 10^{-5} \mathcal{M} B_{\mathrm{ns}, 15} L_{43}^{-1} \Omega_{\mathrm{ns}}^{1 / 2} R_{\mathrm{ns}, 6}^{-3 / 2} v_{9}$.

Making use of equation (60), we find that the photon-electron scattering cross-section, in the strong magnetic field regime $\left(\omega_{\mathrm{B}} \gg\right.$ $\omega)$, when the wave electric field is not exactly perpendicular to the large-scale magnetic field is

$\sigma_{\|} \sim \sigma_{\mathrm{T}} \theta_{\mathrm{kB}}^{2} \sim 2 \times 10^{-45} \mathrm{~cm}^{2} \frac{v_{9}^{2}}{\mathcal{M}^{2} B_{\mathrm{ns}, 15}^{2} \Omega_{\mathrm{ns}}^{2} R_{\mathrm{B}, 8}^{2}}\left(\frac{R}{R_{\mathrm{ns}}}\right)^{6}$,

as long as $\theta_{\mathrm{kB}}>v / \nu_{\mathrm{B}}$, which is the only case we are considering here.

The IC scattering optical depth in this case is

$\tau_{\mathrm{ic}} \approx \frac{3 \sigma_{\|} n L \theta_{\mathrm{s}}^{2}}{64 \pi^{2} R v^{3} m}$ 
where $\theta_{\mathrm{s}}=\min \left\{\ell_{\mathrm{s}} / R, \gamma_{\mathrm{s}}^{-1}\right\}$ is the angular size of the photon beam at $R, \ell_{\mathrm{s}}$ is the transverse size of the FRB source from which photons are received at $R$, and $\gamma_{\mathrm{s}}$ is the LF of the FRB source. Making use of equations (59) and (64), we find the optical depth to IC for $R \lesssim$ $R_{\mathrm{fo}}$ :

$\tau_{\mathrm{ic}} \sim 10^{-7} \frac{L_{43} \ell_{\mathrm{s}, 4}^{2}}{v_{9} R_{\mathrm{B}, 9}^{2} \mathcal{M}_{3} B_{\mathrm{ns}, 15} \Omega_{\mathrm{ns}, 1}}$.

The IC optical depth is less than unity at all radii in the NS magnetosphere at least out to the light cylinder.

Next, we look into particle acceleration and energy loss in the source region of the FRB pulse. In a strong magnetic field region, particle acceleration time for $\mathrm{e}^{ \pm}$plasma due to IC scattering is a slightly modified form of equation (39):

$t_{\mathrm{acc}} \sim \frac{256 \pi^{3} R^{4} \nu^{3} m^{2} c^{2}}{3 \sigma_{\|} \theta_{\mathrm{s}}^{2} L^{2}}$.

Using equation (64) leads to the following expression for the acceleration time:

$t_{\mathrm{acc}} \sim\left(10^{-5} \mathrm{~s}\right) \frac{\nu_{9}\left(B_{\mathrm{ns}, 15} \Omega_{\mathrm{ns}, 1} \mathcal{M}_{3} R_{\mathrm{B}, 9}\right)^{2}}{L_{43}^{2} \ell_{\mathrm{s}, 4}^{2}} \quad$ for $\quad R \lesssim R_{\mathrm{fo}}$.

According to the coherent curvature model of FRBs (Kumar et al. 2017; Lu \& Kumar 2018), $\mathcal{M} \gtrsim 10^{3}$ and $\ell_{\mathrm{s}} \sim 10^{4} \mathrm{~cm}$, and so the IC acceleration time within the source region of FRBs is $\sim 10^{-5}$ $\mathrm{s}$. This is longer than $\ell_{\mathrm{S}} / c$, the residency time of $\mathrm{e}^{ \pm}$in the source region, and hence the IC cannot adversely affect the generation of FRB coherent radiation. In fact, our estimate of $t_{\text {acc }}$ inside the source region is probably too small. This is because the electric field of the radiation is exactly perpendicular to the static magnetic field (Xmode polarization) within the source and $\mathrm{e}^{ \pm}$are stuck in the lowest Landau level and have weaker than classically expected interaction with $\mathrm{X}$-mode photons. It is also the case that the coherent curvature radiation requires a strong electric field along the static magnetic field. Particle acceleration time due to this electric field is of the order of $10^{-16} \mathrm{~s}$ (Kumar et al. 2017), and the electric force on $\mathrm{e}^{ \pm}$ far exceeds any non-zero IC scattering force.

Particles outside the source region, at larger radii, are accelerated to $\mathrm{LF} \sim \theta_{\mathrm{s}}^{-1} \sim R / \ell_{\mathrm{s}} \sim 10^{3} R_{7} / \ell_{\mathrm{s}, 4}$ when $t_{\mathrm{acc}} \ll t_{\mathrm{FRB}} ;$ the FRB radiation becomes roughly isotropic in the particle rest frame when the LF approaches this value, and IC scattering force drops to zero. The total number of $\mathrm{e}^{ \pm}$in the magnetosphere of a magnetar of spin period $1 \mathrm{~s}$ is $\sim 4 \pi \mathcal{M} n_{\mathrm{GJ}} R^{3} \sim 3 \times 10^{36} \mathcal{M}_{3} B_{\mathrm{ns}, 15}$. Therefore, the energy lost by the FRB radiation as it travels through the NS magnetosphere is $\lesssim 2 \times 10^{33} \mathcal{M}_{3}$ erg (isotropic equivalent). This energy loss is a tiny fraction of the total energy of the FRB coherent radiation. ${ }^{8}$

The non-linearity parameter for the FRB coherent radiation (described in Section 2) is $\sim 10^{5}$ at $R=10^{8} \mathrm{~cm}$. In the absence of the magnetic field of the magnetar, $\mathrm{e}^{ \pm}$exposed to this radiation would be accelerated to $L F \sim 10^{10}$. However, the strong magnetic field of a magnetar suppresses particle acceleration drastically, and

\footnotetext{
${ }^{8}$ One might worry that electrons and positrons accelerated to high LF could trigger a pair production avalanche, which can sap the energy from FRB radiation. However, $\mathrm{e}^{ \pm}$accelerated by FRB radiation are moving away from the neutron star, and thus see the NS surface emission highly redshifted and not capable of pair production. Moreover, the photon density associated with pulsar nebula emission is $\lesssim 10 \mathrm{~cm}^{-3}$, which is too small for launching pair cascade.
}

the $\mathrm{LF} \mathrm{e}^{ \pm}$attain at $R=10^{8} \mathrm{~cm}$ is close to unity. ${ }^{9}$ Therefore, particle acceleration by the electric field of FRB radiation does not change the conclusion we arrived at regarding the loss of FRB energy as photons travel through the NS magnetosphere to arrive at Earth; i.e. the loss of energy is negligible.

The main result of this section is that an FRB source located within a few tens of neutron star radii of a magnetar can withstand the enormous radiation forces. Moreover, little energy is lost as the radiation travels though the NS magnetosphere. The reason for this is entirely due to the strong magnetic field of a magnetar that suppresses scattering cross-section and the efficiency of particle acceleration; i.e. in the absence of a strong magnetic field, a large fraction of FRB radiation energy would be imparted to particles along its path as the radiation travels away from the source.

\section{CONCLUSIONS}

We have investigated the effects of an intense FRB coherent radiation on plasma around the region where the radiation is produced. The purpose is to constrain source properties and the radiation mechanism for FRBs by using some fairly general physics considerations, and determine conditions that a successful model should satisfy. In particular, we calculate particle speed due to electric field of the radiation and the highly enhanced scattering (the IC scattering) by electrons and positrons of the coherent FRB radiation where each quantum state has an occupancy number of the order of $10^{35}$. We find that electrons and protons are accelerated to very high LF due to these forces. This severely restricts some, otherwise, promising models for FRBs. One such class of models invokes relativistic shocks and maser-type instability operating in the shock transition zone or downstream of the shock front.

We have shown that the coherent radiation travelling upstream of the shock front stirs up the plasma violently $-\mathrm{e}^{ \pm}$have LF greater than 30 due to the strong electric field of the radiation - even when the shock front is at a distance of $10^{13} \mathrm{~cm}$ from the FRB compact progenitor star (see Section 2). Furthermore, IC scatterings push the upstream plasma away with a large LF as long as the shock front radius is less than $\sim 10^{13} \mathrm{~cm}$, thereby preventing particles from approaching and crossing the shock front to keep the generation of $\mathrm{GHz}$ radiation going.

At larger distances ( $\left.\gtrsim 10^{13} \mathrm{~cm}\right)$, these forces are fairly tame. However, maser-in-shock models require an excessively large amount of energy ( $\gtrsim 10^{46} \mathrm{erg}$ ) in ultrarelativistic outflows to produce a burst at a few $\mathrm{GHz}$ frequency with luminosity $\gtrsim 10^{43} \mathrm{erg} \mathrm{s}^{-1}$, at these large distances (see Section 4.1.1); as a point of reference, the total energy in relativistic outflows in the biggest magnetar flare ever observed (SGR 1806-20) was estimated to be $\sim 10^{44}$ erg from late-time radio observations (Gelfand et al. 2005; Granot et al. 2006). The total energy in relativistic jets for bursts produced by the repeater

\footnotetext{
${ }^{9}$ The angle between the electric field of radiation and the local magnetic field direction minus $\pi / 2$, defined to be $\theta_{\mathrm{kB}}$, is given by equation (60). Thus, the wave non-linearity parameter along the magnetic field $a_{\| 1} \equiv q E \theta_{\mathrm{kB}} /(m c \omega)$ $\ll 1$ for $R \lesssim 10^{8} \mathrm{~cm}$. Therefore, $\mathrm{e}^{ \pm}$are accelerated along the magnetic field by the electric field of the FRB radiation to speeds much smaller than $c$ throughout most of the NS magnetosphere. The acceleration of particles perpendicular to the static magnetic field when the cyclotron frequency $\left(\omega_{\mathrm{B}}\right)$ is much larger than wave frequency $\omega$ is determined by a modified non-linearity parameter $a_{\perp} \equiv q E /\left(m c \omega_{\mathrm{B}}\right)$, which is less than 1 for $R \lesssim 10^{8}$ $\mathrm{cm}$. Therefore, the particle speed perpendicular to the magnetic field is also subrelativistic even quite far from the NS surface; at $R=10^{9} \mathrm{~cm}, a_{\perp} \sim 10$ and $\mathrm{e}^{ \pm} \mathrm{LF}$ is $\sim 10^{2}$.
} 
FRB 121102 in $1 \mathrm{yr}$, for maser models, is required to be substantially larger than $10^{48} \mathrm{erg}$. This exceeds the energy in magnetic fields of an NS with strength of $10^{15} \mathrm{G}$, and that is assuming an efficiency of 100 per cent for converting magnetic energy to relativistic jets; the actual efficiency is perhaps no larger than 1 per cent (Section 4.1.1).

If FRBs were to be associated with SGRs, then that has consequences for the maser-in-shock models. The $\gamma$-ray photons from the SGR will be scattered by electrons upstream of the shock front heating them up. Whether the maser instability can survive this interaction is unclear. Let us consider that the SGR associated with an FRB released $10^{47}$ erg in $\gamma$-rays, and the $\gamma$-ray luminosity was $10^{48} \mathrm{erg} \mathrm{s}^{-1}$; the duration of the $\gamma$-ray pulse for SGR 1806-20 was about $0.1 \mathrm{~s}$. The $\gamma$-ray pulse is ahead of the shock front, when it is at radius $R$, by the distance $\delta R \approx R /\left(2 \gamma_{\mathrm{sw}}^{2}\right) \sim c t_{\mathrm{FRB}}$. Upstream electrons will be heated to the temperature of $\gamma$-rays on a timescale of $t_{\text {ic } \gamma} \sim(1 \mathrm{~ms}) R_{13}^{2} / L_{\gamma, 48}$. Considering this short time-scale, and small Larmor radius $\left(\lesssim 5 \times 10^{3} \mathrm{~cm}\right)$, the upstream particles would have anisotropic velocity distribution as they enter the shock front, and that could affect the instability.

Stimulated Raman and Brillouin scatterings can also attenuate the FRB radiation. The stimulated Raman process has been calculated by many people, e.g. Gangadhara \& Krishan (1992), Thompson et al. (1994), and Levinson \& Blandford (1995). Lyubarsky \& Ostrovska (2016) considered Raman scatterings in the context of stellar corona model for FRBs and concluded that it does not provide more severe constraint on the propagation of FRB radiation than the IC scatterings. However, implications of stimulated Raman and Brillouin scatterings for the more recent models for FRBs need to be investigated.

We have shown in Section 4.2 that the FRB radiation source operating is a region of very strong magnetic field (where the cyclotron frequency is much larger than the FRB radio wave frequency) alleviates these problems; the radiation forces are weaker in the presence of strong magnetic field, and the plasma in the FRB source region is not dispersed quickly. One of the reasons for this is that the interaction cross-section between $\mathrm{e}^{ \pm}$and $\mathrm{X}$-mode photons is highly reduced in the presence of a strong magnetic field. The coherent curvature ('antenna') model for FRBs requires very strong magnetic field for its successful operation (Kumar et al. 2017; Lu \& Kumar 2018). It is shown in Section 4.2 that the source survives the radiative forces, and radiation travelling through the NS magnetosphere suffers negligible loss of energy.

\section{ACKNOWLEDGEMENTS}

We thank the referee, Roger Blandford, for helpful comments and suggestions. WL acknowledges useful discussions with Lorenzo Sironi and Brian Metzger on the physics of synchrotron instability and with Ben Margalit on wind nebulae near magnetars, and we thank Bing Zhang for comments and suggestions. WL was supported by the David and Ellen Lee Fellowship at Caltech.

\section{REFERENCES}

Amiri M. et al., 2019a, Nature, 566, 230

Amiri M. et al., 2019b, Nature, 566, 235
Bannister K. W. et al., 2017, ApJ, 841, L12

Bannister K. W. et al., 2019, Science, 365, 565

Blandford R. D., 1973, A\&A, 26, 161

Blandford R. D., Scharlemann E. T., 1975, Ap\&SS, 36, 303

Blandford R. D., Scharlemann E. T., 1976, MNRAS, 174, 59

Canuto V., Lodenquai J., Ruderman M., 1971, Phys. Rev. D, 3, 2303

Chatterjee S. et al., 2017, Nature, 541, 58

Cordes J. M., Chatterjee S., 2019, ARA\&A, 57, 417

Cordes J. M., Wasserman I., Hessels J. W. T., Lazio T. J. W., Chatterjee S., Wharton R. S., 2017, ApJ, 842, 35

Farah E. L. et al., 2018, MNRAS, 478, 1209

Gaensler B. M. et al., 2005, Nature, 434, 1104

Gajjar V. et al., 2018, ApJ, 863, 2

Gangadhara R. T., Krishan V., 1992, MNRAS, 256, 111

Gelfand J. D. et al., 2005, ApJ, 634, L89

Goldreich P., Julian W. H., 1969, ApJ, 157, 869

Granot J. et al., 2006, ApJ, 638, 391

Granot J., Komissarov S. S., Spitkovsky A., 2011, MNRAS, 411, 1323

Hessels J. W. T. et al., 2019, ApJ, 876, L23

Hurley K. et al., 2005, Nature, 434, 1098

Katz J. I., 2018, Prog. Part. Nucl. Phys., 103, 1

Kennel C. F., Coroniti F. V., 1984, ApJ, 283, 694

Kocz J. et al., 2019, MNRAS, 489, 919

Kumar P., Zhang B., 2015, Phys. Rep., 561, 1

Kumar P., Lu W., Bhattacharya M., 2017, MNRAS, 468, 2726

Law C. J. et al., 2017, ApJ, 850, 76

Levinson A., Blandford R. D., 1995, MNRAS, 274, 717

Lorimer D. R., Bailes M., McLaughlin M. A., Narkevic D. J., Crawford F., 2007, Science, 318, 777

Lu W., Kumar P., 2018, MNRAS, 477, 2470

Lu W., Piro A. L., 2019, ApJ, 883, 40

Lu W., Kumar P., Narayan R., 2019, MNRAS, 483, 359

Luo R., Lee K., Lorimer D. R., Zhang B., 2018, MNRAS, 481, 2320

Lyubarsky Y., 2008, ApJ, 682, 1443

Lyubarsky Y., Ostrovska S., 2016, ApJ, 818, 74

Main R. et al., 2018, Nature, 557, 522

Marcote B. et al., 2017, ApJ, 834, L8

Melrose D. B., 1971, Ap\&SS, 13, 56

Metzger B. D., Berger E., Margalit B., 2017, ApJ, 841, 14

Metzger B. D., Margalit B., Sironi L., 2019, MNRAS, 485, 4091

Michilli D. et al., 2018, Nature, 553, 182

Oslowski S. et al., 2019, MNRAS, 488, 868

Palmer D. M. et al., 2005, Nature, 434, 1107

Petroff E. et al., 2016, Publ. Astron. Soc. Aust., 33, e045

Petroff E., Hessels J. W. T., Lorimer D. R., 2019, A\&AR, 27, 4

Plotnikov I., Sironi L., 2019, MNRAS, 485, 3816

Ravi V., 2019a, MNRAS, 482, 1966

Ravi V., 2019b, Nat. Astron., 3, 928

Ravi V. et al., 2019, Nature, 572, 352

Shannon R. M. et al., 2018, Nature, 562, 386

Spitler L. G. et al., 2014, ApJ, 790, 101

Spitler L. G. et al., 2016, Nature, 531, 202

Tendulkar S. P. et al., 2017, ApJ, 834, L7

Thompson C., Blandford R. D., Evans C., Phinney E. S., 1994, ApJ, 422, 304

Thornton D. et al., 2013, Science, 341, 53

Wilson D. B., Rees M. J., 1978, MNRAS, 185, 297

Yang Y.-P., Zhang B., 2018, ApJ, 868, 31

This paper has been typeset from a $\mathrm{T}_{\mathrm{E}} \mathrm{X} / \mathrm{LT} \mathrm{E} \mathrm{X}$ file prepared by the author. 